CERN-PH-TH-2012-119

DESY $12-078$

HU-EP-12/15

SFB/CPP-12-24

$\mathrm{WUB} / 12-11$

\title{
The strange quark mass and Lambda parameter of two flavor QCD
}

\author{
$\bar{Z}{ }_{\text {collaboration }}^{L P H A}$ \\ Patrick Fritzsch, ${ }^{a}$ Francesco Knechtli, ${ }^{b}$ Björn Leder ${ }^{b}$ Marina Marinkovic, ${ }^{a}$ \\ Stefan Schaefer, ${ }^{c}$ Rainer Sommer ${ }^{d}$ and Francesco Virotta ${ }^{d}$ \\ ${ }^{a}$ Humboldt Universität zu Berlin, Institut für Physik, \\ Newtonstr. 15, 12489 Berlin, Germany \\ ${ }^{b}$ Bergische Universität Wuppertal, \\ Fachbereich C - Mathematik und Naturwissenschaften, \\ Gaussstr. 20, 42119 Wuppertal, Germany \\ ${ }^{c}$ CERN, Physics Department, 1211 Geneva 23, Switzerland \\ ${ }^{d}$ NIC, DESY, Platanenallee 6, 15738 Zeuthen, Germany
}

\begin{abstract}
We complete the non-perturbative calculations of the strange quark mass and the Lambda parameter in two flavor QCD by the ALPHA collaboration. The missing lattice scale is determined via the kaon decay constant, for whose chiral extrapolation complementary strategies are compared. We also give a value for the scale $r_{0}$ in physical units as well as an improved determination of the renormalization constant $Z_{\mathrm{A}}$.
\end{abstract}

Key words: Lattice QCD; Lambda parameter; strange quark mass PACS: 12.38.Gc; 12.38.Aw; 14.65.Bt 


\section{Introduction}

The parameters of the standard model of particle physics have to be determined by matching theory to experimental data. For two flavor QCD we present results for two of these parameters, the scale parameter $\Lambda_{\mathrm{QCD}}$ and the mass of the strange quark.

These results are the outcome of a long project by the ALPHA collaboration. The general strategy using the Schrödinger functional to define the coupling constant has been laid out in Refs. [1,2], and results for the $\Lambda$ parameter in pure gauge theory have been published in [3]. In the two flavor theory for $\Lambda[4,5]$ and the strange quark mass [6], however, the determination of the lattice scale, which allows for their conversion to physical units, has been lacking. This determination is the subject of the present paper.

In lattice computations, the physical mass scale is set by picking one dimensionful observable and identifying its value at the point where the quark mass ratios correspond to the physical situation with the experimental input. If we have done the calculation with all physical effects taken into account (and if the theory is correct), it does not matter, which observable we take. Here, however, we restrict ourselves to QCD with two dynamical flavors of light quarks leading to a systematic uncertainty which is hard to determine. In our computation, we use the kaon decay constant to set the scale. Over the pion decay constant it has the advantage of a chiral extrapolation which is milder and therefore better under control. ${ }^{1}$ However, we need a quenched strange quark. Also the mass of the Omega baryon is popular to set the scale and first results indicate that similar numbers are obtained from this observable [7].

In previous publications the results have been converted to physical units using the scale parameter $r_{0}$ [8], defined via the force between static quarks. The conversion relied on measurements of $r_{0} / a$ by QCDSF [9] and the assumption that $r_{0}=0.5 \mathrm{fm}$. Below, we present our own results for $r_{0} / a$, which differ substantially from the previous values and lead to an update in $r_{0} \Lambda$.

The paper is organized as follows. The lattice action, an overview of the ensembles and details of the error analysis are given in Sect. 2, followed by the definition of the hadronic observables in Sect. 3 and results for the scale parameter $r_{0}$ in Sect. 4. The strategies for the chiral extrapolation are discussed in Sect. 5 leading to the scale determination from the kaon decay constant. The results for the Lambda parameter and the strange quark mass are contained in Sects. 6 and 7 , respectively.

\footnotetext{
${ }^{1}$ This property depends on how one actually approaches the physical point. It is in particular true for the strategy 1 which we introduce below.
} 
The appendices contain updates of many quantities, whose analysis has been subject of previous publications. The renormalization constants $Z_{\mathrm{A}}$ and $Z_{\mathrm{P}}$ are discussed in App. B and App. C, the hadronic scale $L_{1}$ of the Schrödinger functional calculations is subject of App. D, followed by a determination of the critical mass of the improved Wilson fermions and the singlet renormalization factor in App. E.

\section{Lattice parameters and simulation algorithms}

In this computation we use the Wilson plaquette gauge action for the gluon fields together with two degenerate flavors of $\mathrm{O}(a)$ improved Wilson fermions [10]. The action

$$
S[U, \bar{\psi}, \psi]=\beta \sum_{p} \operatorname{tr}[1-U(p)]+a^{4} \sum_{x} \bar{\psi}(x)\left(D+m_{0}\right) \psi(x)
$$

has three parameters: $g_{0}, m_{0}$ and $c_{\mathrm{sw}}$. The coupling constant $g_{0}$ is given by $\beta=6 / g_{0}^{2}$. The fermions with bare mass $m_{0}$, usually substituted by the hopping parameter $\kappa=\left(8+2 a m_{0}\right)^{-1}$, are implemented by the lattice Dirac operator

$$
D=\frac{1}{2}\left\{\gamma_{\mu}\left(\nabla_{\mu}^{*}+\nabla_{\mu}\right)-a \nabla_{\mu}^{*} \nabla_{\mu}\right\}+c_{\mathrm{Sw}} \frac{i a}{4} \sigma_{\mu \nu} \hat{F}_{\mu \nu}
$$

It includes the covariant forward and backward derivatives, $\nabla_{\mu}$ and $\nabla_{\mu}^{*}$, and the Sheikholeslami-Wohlert [11] improvement term involving the standard discretization $\hat{F}_{\mu \nu}$ of the field strength tensor [12]. Its coefficient $c_{\mathrm{sw}}$ has been determined non-perturbatively [13].

We have generated ensembles at three values of $\beta=5.2,5.3$ and 5.5 which correspond roughly to lattice spacings of $a=0.076 \mathrm{fm}, 0.066 \mathrm{fm}$ and $0.049 \mathrm{fm}$, respectively, with details given in Section 5.6. The ensembles are listed in Table 1. All lattices have size $(2 L) \times L^{3}$ and the pion mass is always large enough such that $m_{\pi} L \geq 4$. We therefore expect finite size effects to be small.

\subsection{Simulation algorithms}

For most of the ensembles, generated within the CLS effort, ${ }^{2}$ the DD-HMC algorithm [14,15] has been used as implemented in the software package by M. Lüscher [16]. It is based on a domain decomposition to separate the infrared from the ultraviolet modes of the fermion determinant. A main feature is the locally deflated, Schwarz preconditioned GCR solver $[17,18]$ which significantly reduces the increase in computational cost as the quark mass is lowered.

\footnotetext{
${ }^{2}$ https://twiki.cern.ch/twiki/bin/view/CLS/
} 


\begin{tabular}{cccccccc}
\hline id & $L / a$ & $\beta$ & $\kappa$ & $\kappa_{\mathrm{s}}$ & $R_{0}$ & $m_{\pi}[\mathrm{MeV}]$ & $m_{\pi} L$ \\
\hline A2 & 32 & 5.2 & 0.13565 & $0.135438(20)$ & $5.485(21)$ & 630 & 7.7 \\
A3 & & & 0.13580 & $0.135346(20)$ & $5.674(32)$ & 490 & 6.0 \\
A4 & & & 0.13590 & $0.135285(20)$ & $5.808(34)$ & 380 & 4.7 \\
A5 & & & 0.13594 & $0.135257(20)$ & $5.900(24)$ & 330 & 4.0 \\
\hline E4 & \multirow{2}{*}{32} & 5.3 & 0.13610 & $0.135836(17)$ & - & 580 & 6.2 \\
E5 & & & 0.13625 & $0.135777(17)$ & $6.747(59)$ & 440 & 4.7 \\
F6 & \multirow{2}{*}{48} & & 0.13635 & $0.135741(17)$ & $6.984(51)$ & 310 & 5.0 \\
F7 & & & 0.13638 & $0.135730(17)$ & $7.051(43)$ & 270 & 4.3 \\
\hline N4 & \multirow{2}{*}{48} & 5.5 & 0.13650 & $0.136278(08)$ & $9.32(30)$ & 550 & 6.5 \\
N5 & & & 0.13660 & $0.136262(08)$ & $9.31(26)$ & 440 & 5.2 \\
N6 & & & 0.13667 & $0.136250(08)$ & $9.55(11)$ & 340 & 4.0 \\
O7 & 64 & & 0.13671 & $0.136243(08)$ & $9.68(10)$ & 270 & 4.2 \\
\hline
\end{tabular}

Table 1: Overview of the ensembles used in this study. We give the label, the spatial extent of the lattice, $\beta=6 / g_{0}^{2}$, the hopping parameter $\kappa$ of the sea quarks, the hopping parameter $\kappa_{\mathrm{s}}$ of the strange quark, the scale $R_{0}=r_{0} / a$, the mass of the sea pion $m_{\pi}$ and the product $m_{\pi} L$, which is always larger than or equal to 4 . All lattices have dimension $T \times L^{3}$ with $T=2 L$.

The drawback of this algorithm is that due to the block decomposition only a fraction of gauge links $R_{\text {active }}$ is updated during a trajectory. In pure gauge theory the autocorrelation times are inversely proportional to this fraction of active links [19]; we expect this behavior also in the theory with fermions. Typical domain decompositions lead to active link ratios between 0.37 and 0.5 and therefore a factor between 2 and 3 increased autocorrelation times.

For some lattices, we therefore used a Hybrid Monte Carlo algorithm [20] with a mass preconditioned fermion determinant [21,22]. Our implementation [23], MPHMC from here on, is based on the DD-HMC package and in particular takes over the deflated solver because of its efficient light quark inversions. This algorithm was employed for ensembles A5, N6 and O7 given in Table 1; all other ensembles were generated with the DD-HMC. Appendix A gives details about these algorithms and the values of the parameters used for the gauge field generation.

\subsection{Autocorrelations}

In Monte Carlo data the effect of the autocorrelations has to be accounted for in the error analysis. For all observables $F=F\left(a_{1}, \ldots, a_{n}\right)$, functions of expectation values $a_{i}=\left\langle A_{i}\right\rangle$ of primary observables $A_{i}$, we therefore compute an estimator of 
the autocorrelation function

$$
\Gamma_{F}(t)=\sum_{i, j} f_{i}(\vec{a}) f_{j}(\vec{a})\left\langle\left(A_{i}(t)-a_{i}\right)\left(A_{j}(0)-a_{j}\right)\right\rangle
$$

where $f_{i}=\partial_{i} F(\vec{a})$ following the procedures detailed in Ref. [24]. The argument $t$ indicates the Monte Carlo time. The integrated autocorrelation time is then

$$
\tau_{\text {int }}(F)=\frac{1}{2}+\sum_{t=1}^{\infty} \frac{\Gamma_{F}(t)}{\Gamma_{F}(0)} .
$$

which then enters the statistical error of the observable $\sigma_{F}$ from $N$ measurements

$$
\sigma_{F}^{2}=2 \frac{\tau_{\text {int }}(F)}{N} \Gamma_{F}(0)
$$

The sum in eq. (2.4) is normally truncated at a "window" $W$ [25] which balances the statistical uncertainty due to the limited sample size and the systematic error coming from neglecting the tail for $t>W$. The value of $W$ is determined from the measurement of $\rho_{F}(t)=\Gamma_{F}(t) / \Gamma_{F}(0)$ alone and for each $F$ separately. Neglecting the tail above $W$ leads - at least on average - to an underestimation of $\tau_{\text {int }}$ and the statistical error of the observable. It is particularly problematic in the presence of slow modes of the Monte Carlo transition matrix which only couple weakly to the observable in question. To account for them we use the method outlined in Ref. [19], estimating their time constants from observables to which the slow modes couple strongly. Using them, we can then estimate the tails of the autocorrelation functions of the observables we are interested in and arrive at a more conservative error estimate.

Experience tells us that for small lattice spacing the topological charge is particularly sensitive to slow modes $[19,26]$, for which we use the field theoretical definition after smoothing the field by the Wilson flow integrated up to $t_{0}$ as defined in Ref. [27]. Actually, only the square of the charge needs to be considered, because we are only interested in parity even observables. Unfortunately we are not in the position to accurately determine its autocorrelation time for most of our ensembles. We therefore combine the scaling laws found in pure gauge theory [19] with the measurement for our high statistics ensembles E5 and arrive at the estimate

$$
\tau_{\exp }(\beta)=200 \frac{c_{\tau} e^{7(\beta-5.5)}}{R_{\text {active }}},
$$

in units of molecular dynamics time with $c_{\tau}=2$ for trajectories of length $\tau=0.5$ and $c_{\tau}=1$ for $\tau=2$ and 4 . The values of $R_{\text {active }}$ can be found in Table 9 for the DD-HMC algorithm and is equal to one for the MP-HMC. 
An example of the procedure is given in Fig. 1, showing the autocorrelation function of the kaon decay constant $F_{\mathrm{K}}$ on the $\mathrm{O} 7$ ensemble. Using the standard procedure [24,25], the sum in eq. (2.4) is truncated at the window $W_{l}$ from which we would get $\tau_{\text {int }}=0.7$. When the contribution of the tail is included, the improved estimate gives $\tau_{\text {int }}=4$, which translates to a more than doubled error estimate.

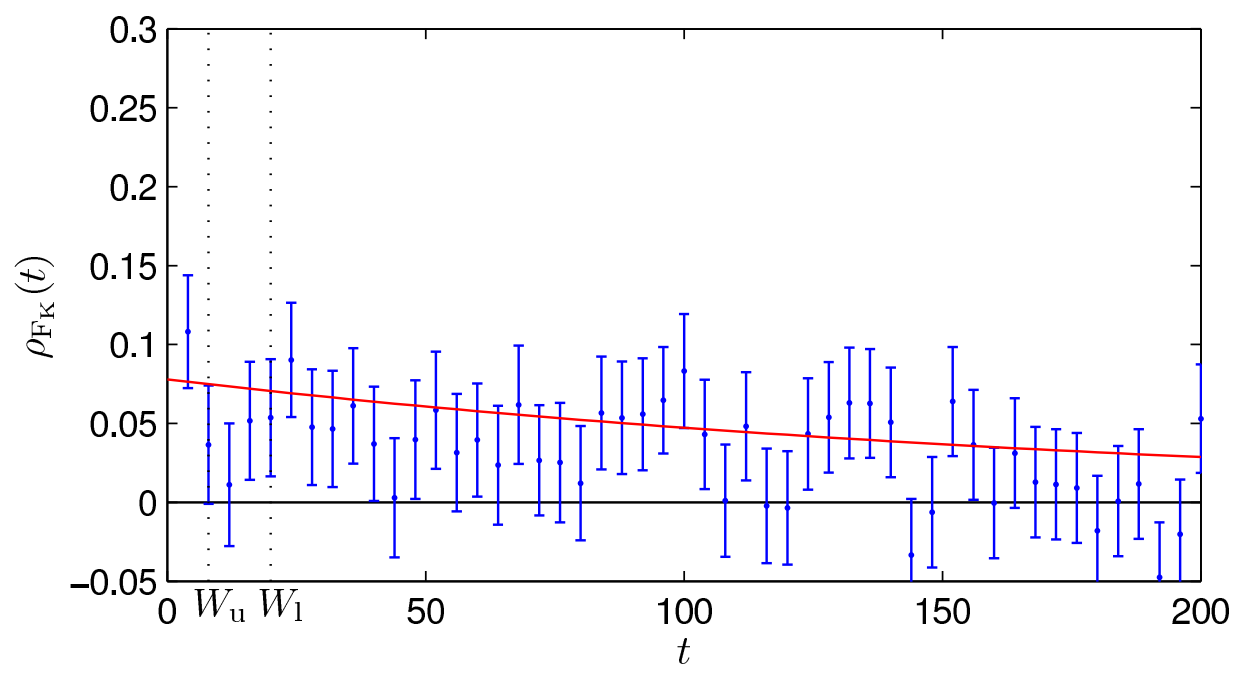

Figure 1: Autocorrelation function of $F_{\mathrm{K}}$ for the $\mathrm{O} 7$ lattice. The line gives our estimate for its tail. The standard method of Ref. [24] gives a window $W=W_{l}$ and $\tau_{\text {int }}=0.7$, compared to $\tau_{\text {int }}=4$ including the tail contribution which we add from $W=W_{u}$, or more than a factor two in the error of the observable.

\section{Observables}

The kaon decay constant necessarily requires the addition of a quenched strange quark to the $N_{\mathrm{f}}=2$ theory. We denote the hopping parameter of this third flavor as $\kappa_{3}$ and have $\kappa_{1}=\kappa_{2}=\kappa_{\text {sea }}$ for the two sea quarks. For the purpose of a definition of the strange quark mass from the PCAC relation, we do in fact add a fourth quenched flavor with $\kappa_{4}=\kappa_{3}$.

The computation of our pseudoscalar observables is based on two-point functions of the pseudoscalar density and the time component of the axialvector cur- 
rent. At a fixed $\kappa_{\text {sea }}$ they are constructed from two valence quarks $r$ and $s$

$$
\begin{aligned}
& f_{\mathrm{PP}}^{r s}\left(x_{0}\right)=-a^{3} \sum_{\vec{x}}\left\langle P^{r s}(x) P^{s r}(0)\right\rangle \\
& f_{\mathrm{AP}}^{r s}\left(x_{0}\right)=-a^{3} \sum_{\vec{x}}\left\langle A_{0}^{r s}(x) P^{s r}(0)\right\rangle
\end{aligned}
$$

with $P^{r s}=\bar{\psi}_{r} \gamma_{5} \psi_{s}$ and $A_{0}^{r s}=\bar{\psi}_{r} \gamma_{0} \gamma_{5} \psi_{s}$. This notation and the analysis that follows is similar to the one presented in Ref. [28]. Using the PCAC relation, average quark masses of flavors $r$ and $s$ can then be defined $a^{3}$

$$
m_{r s}\left(x_{0}\right)=\frac{\frac{1}{2}\left(\partial_{0}+\partial_{0}^{*}\right) f_{\mathrm{AP}}\left(x_{0}\right)+c_{\mathrm{A}} a \partial_{0}^{*} \partial_{0} f_{\mathrm{PP}}\left(x_{0}\right)}{2 f_{\mathrm{PP}}\left(x_{0}\right)} .
$$

In this formula, $\partial_{0}$ and $\partial_{0}^{*}$ denote the forward and backward difference operators in time direction. The improvement coefficient $c_{\mathrm{A}}$ has been determined nonperturbatively [29].

For sufficiently large $x_{0}$ the mass $m_{r s}\left(x_{0}\right)$ will have a plateau over which we can average. From its value $m_{r s}$ the renormalized quark mass $m_{\mathrm{R}}^{r s}$ is obtained [30]

$$
m_{\mathrm{R}}^{r s}=\frac{Z_{\mathrm{A}}\left(1+\bar{b}_{\mathrm{A}} a m_{\mathrm{sea}}+\tilde{b}_{\mathrm{A}} a m_{r s}\right)}{Z_{\mathrm{P}}\left(1+\bar{b}_{\mathrm{P}} a m_{\text {sea }}+\tilde{b}_{\mathrm{P}} a m_{r s}\right)} m_{r s},
$$

with $m_{\text {sea }}=m_{12}$. We will use one-loop perturbation theory for the improvement coefficients $\bar{b}_{\mathrm{A}}, \bar{b}_{\mathrm{P}}, \tilde{b}_{\mathrm{A}}$ and $\tilde{b}_{\mathrm{P}}$, noting that they multiply very small terms. At this order in perturbation theory $\bar{b}_{\mathrm{A}}=\bar{b}_{\mathrm{P}}=0$, while $\tilde{b}_{\mathrm{A}}=1+0.06167 g_{0}^{2}$ and $\tilde{b}_{\mathrm{P}}=1+0.06261 g_{0}^{2}$ computed from the perturbative coefficients of [31]. An update of the non-perturbative determination of $Z_{\mathrm{A}}[32]$ and $Z_{\mathrm{P}}$ [6] is given in App. B and App. C, respectively.

The renormalization and improvement of the PCAC quark masses is much simpler than the corresponding expression in terms of the bare subtracted quark masses $m_{\mathrm{q}, r}=m_{0, r}-m_{\mathrm{cr}}$, where terms proportional to $m_{\mathrm{sea}}$ are present already at the leading order in $a$ [30]. In our analysis we therefore only use the renormalized PCAC relation eq. (3.4). The alternative definition of renormalized quark masses as well as the determination of additive renormalization $m_{\mathrm{cr}}$ and the multiplicative renormalization factor $Z_{m}$ is discussed in App. E.

\subsection{Computation of the two-point functions}

We compute the two-point functions eq. (3.2) using $U(1)$ noise sources $\eta_{t}(x)=$ $\delta_{t, x_{0}} \exp (i \phi(\vec{x}))$ located on randomly chosen time slices $t[33,34]$. Solving the Dirac

\footnotetext{
${ }^{3}$ This definition differs by a factor of two from Ref. [28].
} 
equation once for each noise vector $\zeta_{t}^{r}=Q^{-1}\left(m_{0, r}\right) \eta_{t}=a^{-1}\left(D+m_{0, r}\right)^{-1} \gamma_{5} \eta_{t}$ is sufficient to get an estimator for the two-point functions projected to zero momentum

$$
\begin{aligned}
& a^{3} f_{\mathrm{PP}}^{r s}\left(x_{0}\right)=\sum_{\vec{x}}\left\langle\left[\zeta_{t}^{r}\left(x_{0}+t, \vec{x}\right)\right]^{\dagger} \zeta_{t}^{s}\left(x_{0}+t, \vec{x}\right)\right\rangle, \\
& a^{3} f_{\mathrm{AP}}^{r s}\left(x_{0}\right)=\sum_{\vec{x}}\left\langle\left[\zeta_{t}^{r}\left(x_{0}+t, \vec{x}\right)\right]^{\dagger} \gamma_{0} \zeta_{t}^{s}\left(x_{0}+t, \vec{x}\right)\right\rangle
\end{aligned}
$$

where the average is over noise sources and gauge configurations. For our lattices, we use 10 noise sources per configuration, balancing the numerical cost and the accuracy which we wanted to reach on the given ensembles.

\subsection{Analysis of the data}

The following presentation applies to any flavor combination " $r s$ " and we drop this sub/superscript for the sake of brevity. The mass of the pseudoscalar meson $m_{\mathrm{PS}}$ and its decay constant $f_{\mathrm{PS}}$ can be extracted from $f_{\mathrm{PP}}$ and the PCAC mass. For infinite time extent $T$, the spectral decomposition gives an expansion in terms of functions which decay exponentially for large time separations

$$
f_{\mathrm{PP}}\left(x_{0}\right)=\sum_{i=1}^{\infty} c_{i} e^{-E_{i} x_{0}}
$$

with $E_{1}=m_{\mathrm{PS}}$ the energy of the ground state and $E_{2}<E_{3}<\ldots$ the excited state contribution. For large time separations, we can then extract the decay constant from the leading coefficient

$$
\begin{aligned}
f_{\mathrm{PS}} & =Z_{\mathrm{A}}\left(1+\bar{b}_{\mathrm{A}} a m_{\mathrm{sea}}+a \tilde{b}_{\mathrm{A}} m_{r s}\right) f_{\mathrm{PS}}^{\mathrm{bare}} \\
f_{\mathrm{PS}}^{\mathrm{bare}} & =2 \sqrt{2 c_{1}} m_{r s} m_{\mathrm{PS}}^{-3 / 2} .
\end{aligned}
$$

In the analysis with finite time extent $T$ and time separation $x_{0}$ we have to deal with particles running backwards in time and excited states. Since our lattices are large and the statistical precision of pseudoscalar correlators does not deteriorate at large $x_{0}$, we use the following procedure to fix the region $x_{0} \in$ $\left[x_{0}^{\min }, T-x_{0}^{\min }\right]$, in which we can neglect the excited state contribution: we first perform a fit to the data using the first two terms in the expansion eq. (3.7), now including the finite $T$ effects

$$
f_{\mathrm{PP}}\left(x_{0}\right)=c_{1}\left[e^{-E_{1} x_{0}}+e^{-E_{1}\left(T-x_{0}\right)}\right]+c_{2}\left[e^{-E_{2} x_{0}}+e^{-E_{2}\left(T-x_{0}\right)}\right]
$$

to a range where this function describes the data well, given the accuracy of the data. We then determine $x_{0}^{\mathrm{min}}$ : it is the smallest value of $x_{0}$ where the statistical 


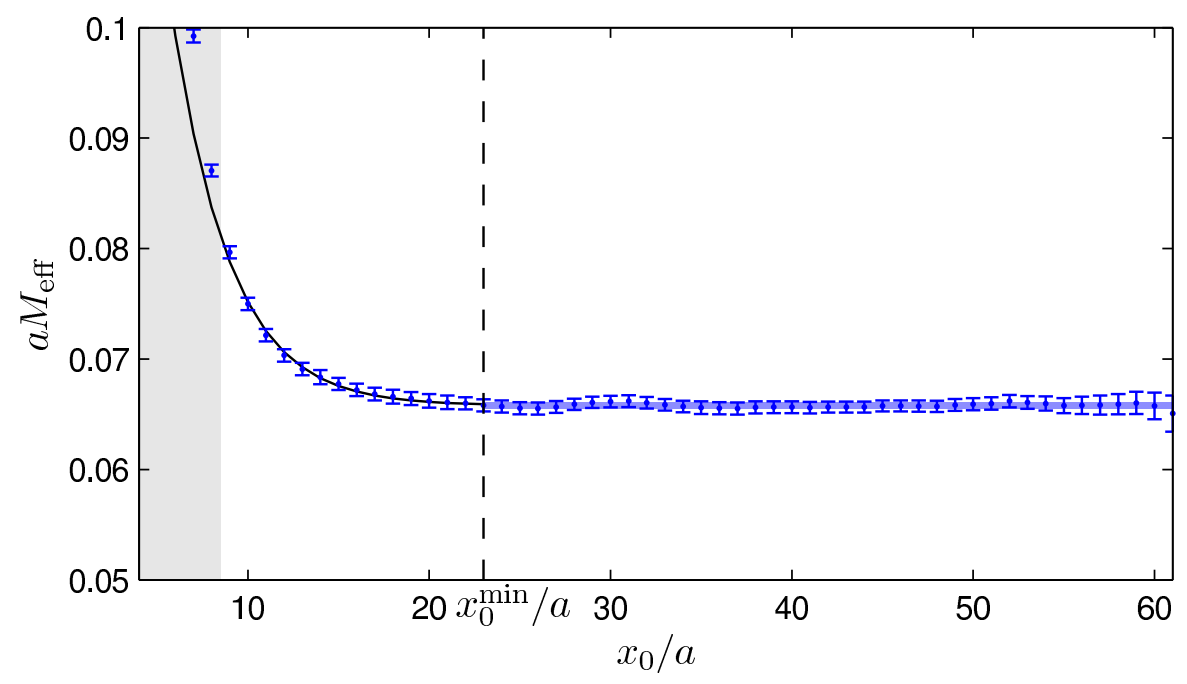

Figure 2: The effective pion mass given by $\cosh \left(M_{\mathrm{eff}}(t-T / 2)\right) / \cosh \left(M_{\mathrm{eff}}(t+1-T / 2)\right)=$ $f_{\mathrm{PP}}(t) / f_{\mathrm{PP}}(t+1)$ for the $\mathrm{O} 7$ lattice. A two-state fit to data outside the shaded area determines $x_{0}^{\min }$. The result of the final one-state fit is given by the error band.

uncertainty on the effective mass is four times larger than the contribution of the excited state as given by the result of the fit using eq. (3.10). In a second step, only the first term of eq. (3.10) is fitted to the data restricted to this region. Figure 2 illustrates the procedure on our largest lattice. Formally at large $x_{0}$ and small sea quark mass the leading correction to eq. (3.7) comes from states which additionally to the ground state have two pions, $E_{2} \approx E_{1}+2 m_{\pi}$. However, for small quark masses and large $L$, the coefficient $c_{2}$ can be computed in chiral perturbation theory. It turns out to be very small [35] (at least for our large volumes) and such a contribution is invisible within our precision. In this sense the value of $E_{2}$ determined by the fit may actually be a higher state, which is one reason for us to use this excited state fit only in order to determine a safe $x_{0}^{\min }$.

The error on the decay constant and the meson mass $m_{\mathrm{PS}}=E_{1}$ are computed including the autocorrelations as described in Sec. 2.2. In appendix F we give the partially quenched meson masses, decay constants and quark masses as well as the values of $x_{0}^{\min }$, which we use in our analysis.

\section{Scale parameter $r_{0}$}

The analysis strategy for the scale $R_{0}(\beta, \kappa)=r_{0} / a$ [8] is based on [36] and we refer to this work for more detailed explanations and notation. The procedure con- 


\begin{tabular}{cccc}
\hline$\beta$ & $n_{1}$ & $n_{2}$ & $n_{3}$ \\
\hline 5.2 & 6 & 9 & 15 \\
5.3 & 8 & 12 & 20 \\
5.5 & 16 & 24 & 40 \\
\hline
\end{tabular}

Table 2: The number of smearing levels $n_{l}$ used to construct the Operators $\hat{O}_{l}, l=1,2,3$ for measuring the Wilson loop correlation matrix.

sists of measuring on-axis Wilson loops $W(r, t)$ on smeared gauge configurations, extracting the static potential $V(r)$ and finally solving the equation

$$
\left.r^{2} F(r)\right|_{r=r_{0}}=1.65
$$

where $F(r)=V^{\prime}(r)$ is the static force. For the latter we use an improved definition which eliminates cut-off effects at tree level. Wilson loops are measured on gauge configurations after all links are replaced by HYP smeared [37] links. We take the HYP2 parameter choice [38] $\alpha_{1}=1.0, \alpha_{2}=1.0$ and $\alpha_{3}=0.5$ and do one HYPsmearing level. The Wilson loops can be exactly represented as an observable in a theory including static quarks and this first smearing step corresponds to the choice of the static quark action (as far as the time-like links are concerned). On the HYP2-smeared gauge link configurations we measure a correlation matrix of Wilson loops $C_{l m}(t)$ for fixed $r$ by smearing the space-like links using numbers $n_{l}$ and $n_{m}$ of spatial HYP smearing iterations. Spatial HYP smearing means that only staples restricted to spatial directions are used and we therefore need only two parameters, which we set to $\alpha_{2}=0.6$ and $\alpha_{3}=0.3$. This second smearing step corresponds in the Hamiltonian formalism to the construction of a variational basis of operators $\hat{O}_{l}$ that create a state consisting of a static quark and antiquark pair. We use a basis of the operators, labelled by $l=1,2,3$. The numbers of smearing iterations at each level $n_{l}$ are listed in Table 2. They are chosen such that the physical extensions of the operators are approximately constant as the lattice spacing changes. Finally we use the generalized eigenvalue method to extract the static potential from the correlation matrix $C_{l m}(t)$, the details of this can be found in [36].

The solution of eq. (4.1) is found by interpolation of the force $F$, using a 2-point interpolation $F(r)=f_{0}+f_{2} / r^{2}$. In order to control the systematic error we compare the result with a 3 -point interpolation adding a $f_{4} / r^{4}$ term. We find the systematic error to be negligible. The error analysis takes into account the coupling to the slow modes as explained in Sect. 2.2 and we neglect the systematic error due to excited state contributions to the potential, because we ensure that 


\begin{tabular}{cclll}
\hline$\beta$ & $S_{2} \equiv 0, c=1.1$ & $S_{2} \equiv 0, c=1.4$ & $c=1.4$ & $c=1.8$ \\
\hline 5.2 & $6.145(59)$ & $6.119(45)$ & $6.22(16)$ & $6.160(79)$ \\
5.3 & $7.259(66)$ & $7.226(47)$ & $7.33(17)$ & $7.274(83)$ \\
5.5 & $10.00(11)$ & $9.953(90)$ & $10.10(26)$ & $10.04(13)$ \\
\hline
\end{tabular}

Table 3: Chiral extrapolations of $R_{0}$ from global fits of the form eq. (4.5). The cut $c$ indicates the upper bound in $x$; the fits with $S_{2} \equiv 0$ do not include a quadratic term in $x$. The final results are taken from the first set.

it is much smaller than the statistical one. The values of $R_{0}$ for each ensemble are listed in Table 1.

In order to define the lattice spacing in a mass independent way we need to perform a chiral extrapolation of $R_{0}$ at fixed $\beta$. The chiral fits are done in the variable

$$
x=\left(r_{0} m_{\mathrm{PS}}\right)^{2} .
$$

Before discussing the chiral fits we study the cut-off effects in the mass dependence of $r_{0}$. For this purpose we define a reference value $r_{0 \text { ref }}$, which corresponds to the value of $r_{0}$ at the pseudoscalar mass

$$
\left.x_{\mathrm{ref}} \equiv\left(r_{0} m_{\mathrm{PS}}\right)^{2}\right|_{\mathrm{ref}}=0.75 .
$$

The reference point eq. (4.3) corresponds to a pseudoscalar mass of $340 \mathrm{MeV}$. In the left plot of Fig. 3, we plot $r_{0} / r_{0 \text { ref }}$ versus $x$ at our three different $\beta$ values. The value of $r_{0 \text { ref }}$ is obtained by linear interpolation and the error analysis of $r_{0} / r_{0 \text { ref }}$ takes into account the correlations between the data. We only consider data with $x \leq 1.4$. Using these data we determine the first coefficient in the Taylor expansion of $r_{0} / r_{0 \text { ref }}(x)$ around $x_{\text {ref }}$ independently for each value of $\beta$

$$
\frac{r_{0}}{r_{0 \text { ref }}}(x)=1+s\left(a / r_{0 \text { ref }}\right) \cdot\left(x-x_{\text {ref }}\right) .
$$

We do not find significant cut-off effects in the slope $s\left(a / r_{0 \text { ref }}\right)[39]$ and in the continuum limit we obtain the value $s(0)=-0.067(10)$ by fitting to a constant.

Motivated by the results in the left plot of Fig. 3, we perform a global fit for all $\beta$ values simultaneously of the form

$$
R_{0}(\beta, x)=R_{0 \mathrm{c}}(\beta)\left(1+S_{1} x+S_{2} x^{2}\right)
$$

and with cuts

$$
x \leq c
$$



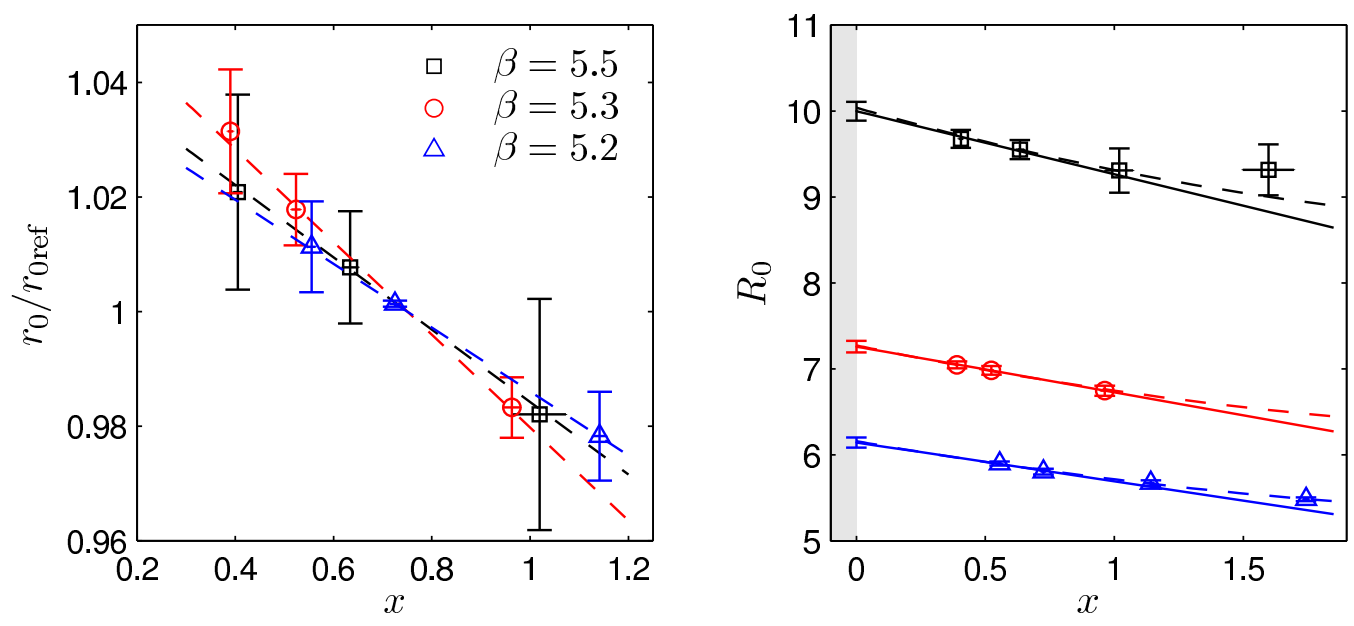

Figure 3: The left plot shows the ratio $r_{0} / r_{0 \text { ref }}$ as a function of $x=\left(r_{0} m_{\mathrm{PS}}\right)^{2}$, where $r_{0 \text { ref }}$ is defined at the reference point eq. (4.3), and the cut $x \leq 1.4$ is applied. The right plot shows our data of $R_{0}=r_{0} / a$ with the chirally extrapolated values using a linear function in $x$ (solid lines) applying the cut $x \leq 1.1$. For comparison a quadratic fit with $c=1.8$ is also shown (dashed lines).

on the pseudoscalar mass. The cuts $c=1.1,1.8$ correspond to $m_{\mathrm{PS}}=410 \mathrm{MeV}$, $530 \mathrm{MeV}$. The fit takes into account both errors on $R_{0}$ and on $x$. The chirally extrapolated values $R_{0 \mathrm{c}}(\beta)$ are listed in Table 3 for various possibilities of the global fit. Columns two and three are linear fits while columns four and five are quadratic fits. We quote as our final numbers for $R_{0 c}(\beta)$ the results in the second column of Table 3 from the linear fit with $c=1.1$ (which has $\chi^{2} /$ dof $=0.07$ and $\left.S_{1}=-0.073(14)\right)$ :

$$
R_{0 \mathrm{c}}(5.2)=6.145(59), \quad R_{0 \mathrm{c}}(5.3)=7.259(66), \quad R_{0 \mathrm{c}}(5.5)=10.00(11) .
$$

The data for $R_{0}(\beta, x)$ and the linear fit with $c=1.1$ (solid lines) are shown in the right plot of Fig. 3. The numbers in eq. (4.7) cover all the fit results of Table 3 within errors. In particular they are perfectly consistent with the results in the fifth column of Table 3 from a quadratic fit applying the cut $c=1.8$ (which has $\chi^{2} /$ dof $=0.28, S_{1}=-0.085(25)$ and $S_{2}=0.013(11)$ ). This quadratic fit is represented by dashed lines in the right plot of Fig. 3. The quadratic term is not significant even with $c=1.8$.

The discrepancy with the determination of $r_{0}$ by QCDSF [40] was discussed in [41]. Meanwhile QCDSF updated their values, ${ }^{4}$ which now agree with our determination where they can be compared.

\footnotetext{
${ }^{4} \mathrm{G}$. Bali, private communication.
} 


\section{Chiral extrapolation of $f_{\mathrm{K}}$ and strange quark mass}

In this section we describe our central determination of the scale. For a number of reasons, it is based on the kaon decay constant $f_{\mathrm{K}}$. First, $f_{\mathrm{K}}$ is experimentally accessible once the CKM-matrix element $V_{\text {us }}$ is considered known, which is a good assumption within the envisaged precision. Second, chiral perturbation theory (ChPT) provides a theory for the quark mass dependence of $f_{\mathrm{K}}$ at small masses of the light quarks, i.e., our sea quarks. Third, as already mentioned, we remain within the pseudoscalar sector of the theory, where ground state properties can be determined without doubt.

\subsection{Our strategies}

The main difficulty and source of a systematic error is the extrapolation to the proper quark masses, the "physical point". Once we decide to set the scale through $f_{\mathrm{K}}$, this point is naturally defined by

$$
R_{\mathrm{K}}=R_{\mathrm{K}}^{\mathrm{phys}}, \quad R_{\pi}=R_{\pi}^{\mathrm{phys}}
$$

where

$$
R_{\mathrm{K}}=\frac{m_{\mathrm{K}}^{2}}{f_{\mathrm{K}}^{2}}, \quad R_{\pi}=\frac{m_{\pi}^{2}}{f_{\mathrm{K}}^{2}},
$$

and $R_{\mathrm{K}}^{\text {phys }}, R_{\pi}^{\text {phys }}$ are the values of these ratios in Nature. In an attempt to minimize uncertainties, we take the physical masses and decay constants to be the ones in the isospin symmetric limit with QED effects removed as discussed in [42]. We use

$$
m_{\pi, \text { phys }}=134.8 \mathrm{MeV}, \quad m_{\mathrm{K}, \mathrm{phys}}=494.2 \mathrm{MeV}, \quad f_{\mathrm{K}, \mathrm{phys}}=155 \mathrm{MeV} .
$$

The two conditions eq. (5.1) define a point in the plane spanned by $m_{\text {light }}, m_{\mathrm{s}}$, or equivalently $\kappa_{1}, \kappa_{3}$. We are presently not able to simulate at or very close to this physical point, especially not for the smallest lattice spacing, where huge lattices would be needed to keep finite size effects under control. The physical point has to be approached from unphysically large values of $m_{\text {light }}$. Along which trajectory in the plane of bare parameters one approaches the physical point is in principle arbitrary. However, one would like the quantity that is to be computed - here $f_{\mathrm{K}}$ - to depend very little on the distance to the physical point, allowing for an easy extrapolation. Secondly, one would like a theoretically motivated extrapolation formula. In ChPT both $m_{\mathrm{K}}$ and $f_{\mathrm{K}}$ depend on the sum of the quark masses at leading order in the systematic expansion in small quark masses. Keeping

$$
R_{\mathrm{K}}\left(\kappa_{1}, \kappa_{3}\right)=R_{\mathrm{K}}^{\mathrm{phys}}
$$



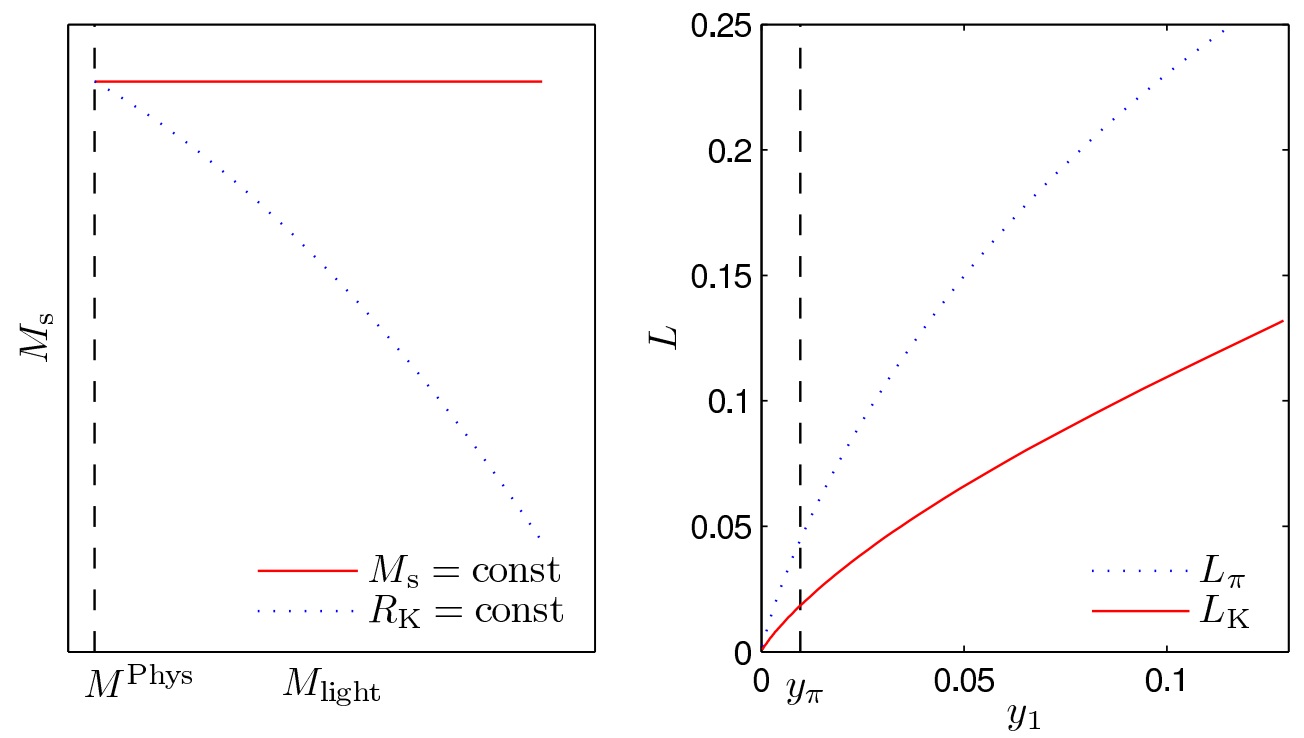

Figure 4: Left: Trajectories to approach the physical point in the plane of light and strange quark mass $M_{\text {light }}, M_{\mathrm{s}}$. The dotted line corresponds to strategy 1, i.e., $R_{\mathrm{K}}=R_{\mathrm{K}}^{\text {phys }}$, whereas strategy 2 (full line) holds $M_{\mathrm{s}}$ fixed. Right: The two functions $L_{\mathrm{K}}$ and $L_{\pi}$, eqs. (5.9) and (5.13) respectively, in the interval $y_{1} \in\left[0, y_{\mathrm{K}}\right]$.

thus defines a trajectory where $f_{\mathrm{K}}$ varies little in ChPT. We will discuss this quantitatively below. An additional advantage is that all along this trajectory we have $m_{\mathrm{K}} \approx m_{\mathrm{K}}^{\text {phys }}$, while for a more conventional trajectory, where $m_{\mathrm{s}}$ is kept constant, the mass $m_{\mathrm{K}}$ is significantly heavier than $m_{\mathrm{K}}^{\mathrm{phys}}$. Since the ChPT expansion is written in terms of $m_{\mathrm{K}}^{2}$ and $m_{\pi}^{2}$, having $m_{\mathrm{K}}$ no larger then $m_{\mathrm{K}}^{\text {phys }}$ increases our chance of being inside the expansion's domain of applicability. To our knowledge this strategy has not been used so far, somewhat surprisingly.

As an alternative we use a second strategy, where we keep $m_{\mathrm{s}}$ constant, however instead of using the expansion in $m_{\mathrm{K}}^{2}$ and $m_{\pi}^{2}$, we use $\mathrm{SU}(2) \mathrm{ChPT}$ for kaon observables [43,44], where only $m_{\pi}^{2}$ is considered a small parameter (in units of the chiral scale $4 \pi f$ ). This strategy provides a suitable definition for future investigations of mesons and baryons with strange quark content.

The trajectories belonging to the two strategies, whose details are the subject of the following sections, are schematically shown in Fig. 4 on the left.

Since the following section deals with the scale setting, we want to distinguish between the kaon decay constant in physical units $f_{\mathrm{K}}$ and in lattice units $F_{\mathrm{K}}$

$$
F_{\mathrm{K}} \equiv a f_{\mathrm{K}} .
$$




\subsection{Strategy 1 and partially quenched SU(3) ChPT}

As discussed above, the trajectory $\kappa_{3}=h\left(\kappa_{1}\right)$ is defined by

$$
R_{\mathrm{K}}\left(\kappa_{1}, h\left(\kappa_{1}\right)\right)=R_{\mathrm{K}}^{\text {phys }},
$$

where $R_{\mathrm{K}}$ is considered a function $R_{\mathrm{K}}\left(\kappa_{1}, \kappa_{3}\right)$. In practice we take a fixed sea quark hopping parameter $\kappa_{1}$ and a few values of $\kappa_{3}$ for which $R_{\mathrm{K}}\left(\kappa_{1}, \kappa_{3}\right)$ is close to $R_{\mathrm{K}}^{\text {phys }}$ and then interpolate in $\kappa_{3}$ to find $h\left(\kappa_{1}\right)$. Also $f_{\mathrm{K}}\left(\kappa_{1}, \kappa_{3}\right)$ is interpolated to $\kappa_{3}=h\left(\kappa_{1}\right)$. We give more details on the various interpolations in $\kappa_{3}$ in Sect. 5.4.

It remains to extrapolate $f_{\mathrm{K}}\left(\kappa_{1}, h\left(\kappa_{1}\right)\right)$ in $\kappa_{1}$ to the physical point eq. (5.1). We use the partially quenched chiral perturbation theory results by Sharpe [45], implement our condition eq. (5.4), which expresses $m_{\mathrm{K}}$ in terms of $m_{\pi}$, and find

$$
\begin{aligned}
f_{\mathrm{K}}\left(\kappa_{1}, h\left(\kappa_{1}\right)\right) & =f_{\mathrm{K}, \text { phys }}\left[1+\bar{L}_{\mathrm{K}}\left(y_{1}, y_{\mathrm{K}}\right)+\left(\alpha_{4}-\frac{1}{4}\right)\left(y_{1}-y_{\pi}\right)+\mathrm{O}\left(y^{2}\right)\right], \\
\bar{L}_{\mathrm{K}}\left(y_{1}, y_{\mathrm{K}}\right) & =L_{\mathrm{K}}\left(y_{1}, y_{\mathrm{K}}\right)-L_{\mathrm{K}}\left(y_{\pi}, y_{\mathrm{K}}\right), \\
L_{\mathrm{K}}\left(y_{1}, y_{\mathrm{K}}\right) & =-\frac{1}{2} y_{1} \log \left(y_{1}\right)-\frac{1}{8} y_{1} \log \left(2 y_{\mathrm{K}} / y_{1}-1\right) .
\end{aligned}
$$

The variables $y_{i}$ are proportional to (averages of) quark masses up to quadratic terms

$$
\begin{aligned}
& y_{1}=\frac{m_{\pi}^{2}\left(\kappa_{1}\right)}{8 \pi^{2} f_{\mathrm{K}}^{2}\left(\kappa_{1}, h\left(\kappa_{1}\right)\right)}, \\
& y_{\pi}=\frac{m_{\pi, \text { phys }}^{2}}{8 \pi^{2} f_{\mathrm{K}, \mathrm{phys}}^{2}}=0.00958, \quad y_{\mathrm{K}}=\frac{m_{\mathrm{K}, \mathrm{phys}}^{2}}{8 \pi^{2} f_{\mathrm{K}, \mathrm{phys}}^{2}}=0.12875 .
\end{aligned}
$$

Because of eq. (5.4), we have $y_{3} \equiv m_{\mathrm{K}}^{2}\left(\kappa_{1}, h\left(\kappa_{1}\right)\right) /\left[8 \pi^{2} f_{\mathrm{K}}^{2}\left(\kappa_{1}, h\left(\kappa_{1}\right)\right)\right]=2 y_{\mathrm{K}}-y_{1}+$ $\mathrm{O}\left(y^{2}\right)$ and $y_{3}$ does not appear in eq. (5.7).

At this order in the chiral expansion we can also replace

$$
y_{1} \rightarrow \tilde{y}_{1}=\frac{m_{\pi}^{2}\left(\kappa_{1}\right)}{8 \pi^{2} f_{\pi}^{2}\left(\kappa_{1}\right)},
$$

with the corresponding replacement $y_{\pi} \rightarrow \tilde{y}_{\pi}$, which we will use as a check of the typical size of $\mathrm{O}\left(y^{2}\right)$ effects.

In the right panel of Fig. 4 we compare the chiral $\log$ function $L_{\mathrm{K}}$ to the one describing the chiral behavior of $f_{\pi}$,

$$
\begin{aligned}
& f_{\pi}\left(\kappa_{1}\right)=f_{\pi, \text { phys }}\left[1+\bar{L}_{\pi}\left(y_{1}\right)+\left(\alpha_{4}+\frac{1}{2} \alpha_{5}\right)\left(y_{1}-y_{\pi}\right)+\mathrm{O}\left(y_{1}^{2}\right)\right], \\
& L_{\pi}\left(y_{1}\right)=-y_{1} \log \left(y_{1}\right), \quad \bar{L}_{\pi}\left(y_{1}\right)=L_{\pi}\left(y_{1}\right)-L_{\pi}\left(y_{\pi}\right) .
\end{aligned}
$$


Our condition eq. (5.6) leads to the specific combination of chiral logarithms eq. (5.9), which has very little curvature and is overall much smaller than $L_{\pi}$; the suppression of the light quark mass dependence thus extends also to the NLO chiral logarithms. This suggests that the chiral extrapolation is much easier than for $f_{\pi}$ and was one of our reasons to select $f_{\mathrm{K}}$ to set the scale. Of course, the counter terms $\alpha_{i}$ do not contribute in Fig. 4 (right), but as they are linear in $y_{1}$ introduce no curvature.

\subsection{Strategy 2 and $\mathrm{SU}(2) \mathrm{ChPT}$}

Here we extrapolate in the light quark mass at fixed mass of the strange quark, namely we tune for each sea quark mass the strange quark's hopping parameter such that the PCAC mass $a m_{34}$ has a prescribed value $\mu$, which is independent of $\kappa_{1} \cdot{ }^{5}$ This defines the function $\kappa_{3}=s\left(\kappa_{1}, \mu\right)$. In practice, we again interpolate the data for $m_{34}\left(\kappa_{1}, \kappa_{3}\right)$ in $\kappa_{3}$ and then solve

$$
\operatorname{am}_{34}\left(\kappa_{1}, s\left(\kappa_{1}, \mu\right)\right)=\mu
$$

for $s$, with the left hand side represented by the interpolation formula.

To find the value of $\mu$ corresponding to the physical point, we employ $\mathrm{SU}(2)$ ChPT $[43,44]$ to first extrapolate $M_{\mathrm{K}}^{2}=\left(a m_{\mathrm{K}}\right)^{2}$ and $F_{\mathrm{K}}$ (both interpolated in $\kappa_{3}$ to the point $\left.\kappa_{3}=s\left(\kappa_{1}, \mu\right)\right)$ in $y_{1}$ to $y_{1}=y_{\pi}$ at fixed value of $\mu$,

$$
F_{\mathrm{K}}\left(\kappa_{1}, s\left(\kappa_{1}, \mu\right)\right)=p(\mu)\left[1-\frac{3}{8}\left[y_{1} \log \left(y_{1}\right)-y_{\pi} \log \left(y_{\pi}\right)\right]+\alpha_{\mathrm{f}}(\mu)\left(y_{1}-y_{\pi}\right)+\mathrm{O}\left(y_{1}^{2}\right)\right]
$$

$M_{\mathrm{K}}^{2}\left(\kappa_{1}, s\left(\kappa_{1}, \mu\right)\right)=q(\mu)\left[1+\alpha_{\mathrm{m}}(\mu)\left(y_{1}-y_{\pi}\right)+\mathrm{O}\left(y_{1}^{2}\right)\right]$.

These expressions represent the asymptotic expansions for small $y_{1}$ at fixed $\mu$ correct up to error terms of order $y_{1}^{2}{ }^{6}$

From eq. (5.15), $q(\mu)$ and $p(\mu)$ are computable for arbitrary values of $\mu$. The requirement that $m_{\mathrm{K}}^{2} / f_{\mathrm{K}}^{2}$ attains its physical value at the physical light quark mass then defines $\mu_{\mathrm{s}}$,

$$
\frac{q\left(\mu_{\mathrm{s}}\right)}{p\left(\mu_{\mathrm{s}}\right)^{2}}=\frac{m_{\mathrm{K}, \mathrm{phys}}^{2}}{f_{\mathrm{K}, \mathrm{phys}}^{2}} .
$$

\footnotetext{
${ }^{5}$ In principle we should keep $m_{\mathrm{R}}^{34}$ fixed, but the ratio $m_{\mathrm{R}}^{34} / m_{34}$ is independent of $\kappa_{1}$ since we set $\bar{b}_{\mathrm{A}}=\bar{b}_{\mathrm{P}}=0$, see eq. (3.4).

${ }^{6}$ We note that $\mathrm{SU}(2) \mathrm{ChPT}$ for kaons does not take into account kaon loops as opposed to $\mathrm{SU}(3) \mathrm{ChPT}$. This corresponds to the production of two kaons, i.e., states with an energy of around one GeV. From this point of view it is not really worse than ChPT in the pion sector where $|\rho \pi\rangle$ intermediate states are dropped. We thank Gilberto Colangelo for emphasizing this point.
} 
This equation is solved numerically for $\mu_{\mathrm{s}}$ and the lattice spacing is then given by

$$
a=\frac{p\left(\mu_{\mathrm{s}}\right)}{f_{\mathrm{K}, \mathrm{phys}}} .
$$

As before, the constants $\alpha_{\mathrm{f}}\left(\mu_{\mathrm{s}}\right), \alpha_{\mathrm{m}}\left(\mu_{\mathrm{s}}\right)$ have common values for all three $\beta$ in the fits to eq. (5.15).

\subsection{Interpolations}

In various places we need observables, such as $R_{\mathrm{K}}\left(\kappa_{1}, \kappa_{3}\right)$ as a continuous function of $\kappa_{3}$, not just for a few numerical values. In all cases we chose four different numerical values for $\kappa_{3}$, close to the required one, namely those which have the smallest distance defined by $d=\left|R_{\mathrm{K}}\left(\kappa_{1}, \kappa_{3}\right)-R_{\mathrm{K}}^{\text {phys }}\right|$. We then determine an interpolation polynomial $P_{2}\left(\kappa_{3}-\kappa_{1}\right)=\sum_{n=0}^{2} c_{n}\left(\kappa_{3}-\kappa_{1}\right)^{n}$ through a fit to the data with weights $\left(d^{2}+\epsilon^{2}\right)^{-1}$. The regulating term $\epsilon$ is chosen as $\epsilon=\frac{1}{100}\left(\max _{\kappa_{3}}\left\{R_{\mathrm{K}}\left(\kappa_{1}, \kappa_{3}\right)\right\}-\min _{\kappa_{3}}\left\{R_{\mathrm{K}}\left(\kappa_{1}, \kappa_{3}\right)\right\}\right)$. We always checked that uncertainties due to the specific interpolation are negligible by considering natural variants.

\subsection{Cutoff effects}

So far the discussion neglects lattice artifacts completely. We will see that these are very small in our formulation. Since both $y_{1}$ and $a^{2}$ are small, it is natural to keep terms of order $y_{1}$ (as done above) and those of order $a^{2}$ which are independent of $y_{1}$ but drop terms of order $y_{1}^{2}, y_{1} a^{2}, a^{3}$ etc. In this approximation we can use eq. (5.7) for the decay constants in lattice units $F_{\mathrm{K}}$, and with a global, $a$-independent low energy constant $\alpha_{4}$. The straight $a^{2}$ term does not contribute to eq. (5.7), since $f_{\mathrm{K} \text {,phys }}$ is used to set the scale. Such a term is, however, present in general. An example is the combination $r_{0} f_{\mathrm{K}}$. It is shown in Fig. 5 with open symbols, where both $f_{\mathrm{K}}$ and $r_{0}$ are evaluated at the finite quark mass. Since a ChPT expansion of $r_{0}$ does not exist, we use

$$
R_{0}\left(\kappa_{1}\right) F_{\mathrm{K}}\left(\kappa_{1}, h\left(\kappa_{1}\right)\right)=\left[\left.r_{0}\right|_{y=y_{\pi}} f_{\mathrm{K}, \mathrm{phys}}\right]_{\mathrm{cont}}\left[1+A\left(y_{1}-y_{\pi}\right)+B F_{\mathrm{K}}^{2}\right]
$$

to describe the data and to extrapolate to the physical point and continuum limit $\left[\left.r_{0}\right|_{y=y_{\pi}} f_{\mathrm{K}, \mathrm{phys}}\right]_{\text {cont }}$. Taking into account that we have $F_{\mathrm{K}}^{2}<4 \cdot 10^{-3}$, the lattice artifact parametrized by $B$ is rather small, see Table 4 . In these fits we take the chirally extrapolated value $F_{\mathrm{K}}$ in the last term of eq. (5.18). Replacing it with $F_{\mathrm{K}}$ at the given value of $y_{1}$, we get an insignificant shift of $0.6 \%$ in the continuum value, which tests the smallness of $y_{1} a^{2}$ terms. 


\begin{tabular}{ccccccc}
\hline$y_{1}^{\max }$ & $\left.r_{0}\right|_{y=y_{\pi}} f_{\mathrm{K}, \mathrm{phys}}$ & $A$ & $B$ & $r_{0 \mathrm{c}} f_{\mathrm{K}, \mathrm{phys}}$ & $\alpha_{4}$ & $B^{\prime}$ \\
\hline 0.10 & $0.3951(62)$ & $0.28(22)$ & $-20(5)$ & $0.3950(75)$ & $0.52(11)$ & $-22(6)$ \\
\hline
\end{tabular}

Table 4: Results for chiral fits in the range $y_{1} \leq y_{1}^{\max }$.

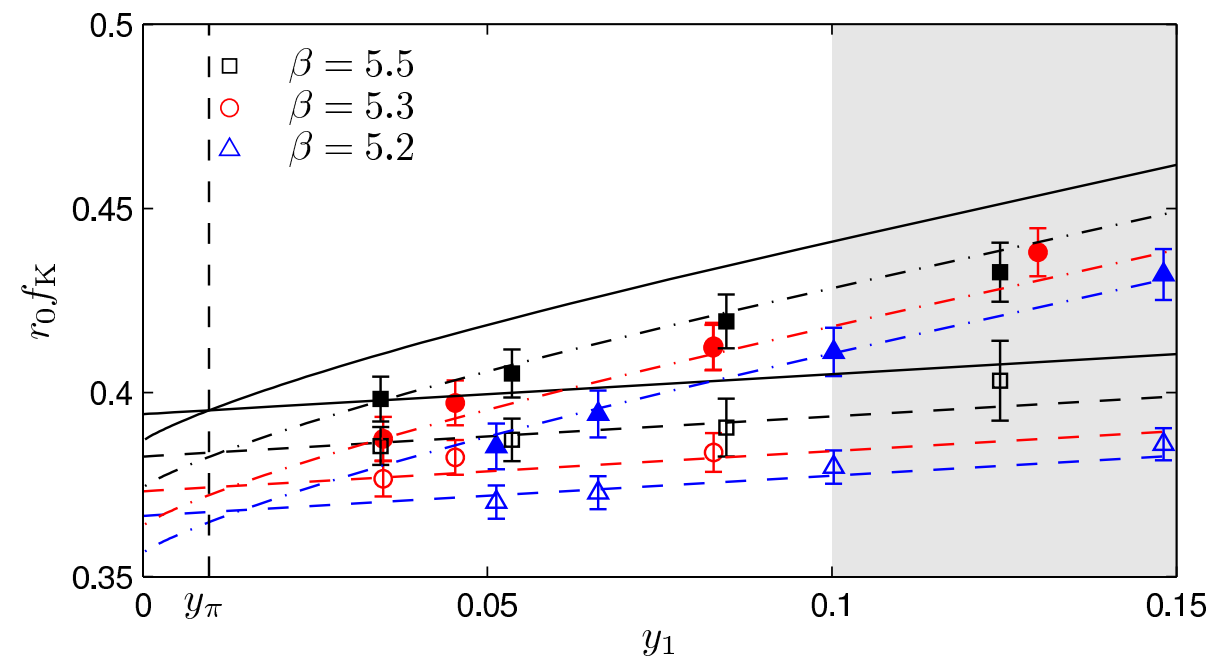

Figure 5: Chiral extrapolation of $r_{0} f_{\mathrm{K}}$. For the open symbols $r_{0}$ and $f_{\mathrm{K}}$ are evaluated at finite quark mass, the dashed lines show eq. (5.18) for the three values of $\beta$. The filled symbols use the extrapolated value $r_{0}=r_{0 c}$, the dashed dotted lines represent eq. (5.19). In both cases, the corresponding solid line gives the continuum result.

Alternatively, we use the already chirally extrapolated values $R_{0 \mathrm{c}}$ from Sect. 4 and

$$
\begin{aligned}
& R_{0 \mathrm{c}} F_{\mathrm{K}}\left(\kappa_{1}, h\left(\kappa_{1}\right)\right) \\
& \quad=\left[r_{0 \mathrm{c}} f_{\mathrm{K}, \mathrm{phys}}\right]_{\mathrm{cont}}\left[1+\bar{L}_{\mathrm{K}}\left(y_{1}, y_{\mathrm{K}}\right)+\left(\alpha_{4}-\frac{1}{4}\right)\left(y_{1}-y_{\pi}\right)+B^{\prime} F_{\mathrm{K}}^{2}\right] .
\end{aligned}
$$

The dashed dotted lines in Fig. 5 show the corresponding fit with the parameters listed in Table 4.

The just discussed fits mainly illustrate that cut-off effects are small and provide a motivation that indeed terms such as $a^{2} y_{1}$ can be dropped. We then determine the values of the kaon decay constant in lattice units at the physical point $F_{\mathrm{K} \text {,phys }}$ by a direct application of

$$
F_{\mathrm{K}}\left(\kappa_{1}, h\left(\kappa_{1}\right)\right)=F_{\mathrm{K}, \mathrm{phys}}\left[1+\bar{L}_{\mathrm{K}}\left(y_{1}, y_{\mathrm{K}}\right)+\left(\alpha_{4}-\frac{1}{4}\right)\left(y_{1}-y_{\pi}\right)\right] .
$$

Here the data at the three different $\beta$ are combined in a global fit with one common 
value of $\alpha_{4}$, but, of course, with different $F_{\mathrm{K} \text {,phys }}$ for the three different $\beta$. The lattice spacings are then obtained from

$$
a=\frac{F_{\mathrm{K}, \mathrm{phys}}}{f_{\mathrm{K}, \mathrm{phys}}} .
$$

Results are discussed in Sect. 5.6 together with our alternative strategy.

We finish the discussion of cut-off effects by mentioning an investigation of the PCAC relation. In a properly $\mathrm{O}(a)$ improved theory we have $\Delta=\frac{1}{2}\left(m_{\mathrm{R}}^{12}+\right.$ $\left.m_{\mathrm{R}}^{34}\right)-m_{\mathrm{R}}^{13}=\mathrm{O}\left(a^{2}\right)$. We have computed $\Delta$ for all our combinations of $\beta, \kappa_{1}, \kappa_{3}$ and found $a \Delta<10^{-4}$ and $\Delta<0.3 \mathrm{MeV}$ in physical units. ${ }^{7}$ This confirms again the smallness of cut-off effects.

\subsection{Numerical results}

We now apply the above formulae to a determination of the kaon decay constant and the strange quark mass. The renormalization of the decay constant, eq. (3.8), starts from $Z_{\mathrm{A}}$ computed in [32] and improved in statistical precision in App. B. For the mass-dependent $\mathrm{O}(a)$ improvement terms, which yield very small corrections, we use $\bar{b}_{\mathrm{A}}=0$ and $\tilde{b}_{\mathrm{A}}=1+0.06167 g_{0}^{2}$ from one-loop perturbation theory [31]. The extrapolations of $F_{\mathrm{K}}$ through eq. (5.20), strategy 1 , and eq. (5.15), strategy 2, are shown in Fig. 6. In the case of strategy 2, the parameter $\mu$ is fixed to the strange quark mass $\mu=\mu_{\mathrm{s}}$ through eq. (5.16), with the values of $\kappa_{\mathrm{s}}$ given in Table 1 . The fits include data for $y_{1}<y_{1}^{\max }$ with $y_{1}^{\max }=0.1$. We observe that the two rather different chiral effective theory extrapolations yield results in close agreement at the physical point. Table 5 lists the fit parameters and also includes fits with $y_{1} \rightarrow \tilde{y}_{1}$, simple linear fits in $y_{1}$ and $\tilde{y}_{1}$ as well as results including data out to $y_{1}^{\max }=0.15$.

A few observations are worth pointing out. Using $\tilde{y}_{1}$ as the chiral variable leads to a stronger dependence on the cut $y_{1}^{\max }$. Still, the extrapolation in terms of $\tilde{y}_{1}$ shows a tendency to converge to the one in terms of $y_{1}$ when the cut is lowered, as it should be. Results of linear extrapolations are also very close.

We therefore take our central values from the results of strategy 1 with cut $y_{1}^{\max }=0.1$. As a systematic error we take into account the deviations to the fit following strategy 2 and to a simple linear extrapolation; for our smallest lattice spacing these different extrapolations are compared more closely in Fig. 7. The final numbers for $F_{\mathrm{K}}$ at the physical point and the associated lattice spacings are shown in Table 6.

\footnotetext{
${ }^{7}$ The improvement term proportional to $\tilde{b}_{\mathrm{A}}-\tilde{b}_{\mathrm{P}}=\left(b_{\mathrm{A}}-b_{\mathrm{P}}\right) / Z$ was inserted nonperturbatively using $b_{\mathrm{A}}-b_{\mathrm{P}}$ and $Z$ from [46]. It is very small in practice.
} 


\begin{tabular}{|c|c|c|c|c|c|c|c|c|}
\hline fit & $y_{1}^{\max }$ & $\beta$ & & fits with & & & fits with? & \\
\hline & ategy & & $10^{2} F_{\mathrm{K}}$ & $\alpha_{4}$ & $10^{2} a[\mathrm{fm}]$ & $10^{2} F_{\mathrm{K}}$ & $\alpha_{4}$ & $10^{2} a[\mathrm{fm}]$ \\
\hline$(5.20)$ & 0.15 & 5.2 & $5.92(7)$ & $0.67(6)$ & $7.53(9)$ & $5.81(7)$ & $0.98(7)$ & $7.40(9)$ \\
\hline & & 5.3 & $5.15(5)$ & & $6.55(7)$ & $5.05(5)$ & & $6.43(7)$ \\
\hline & & 5.5 & $3.80(3)$ & & $4.84(4)$ & $3.73(3)$ & & $4.75(4)$ \\
\hline & 0.1 & 5.2 & $5.93(7)$ & $0.57(12)$ & $7.55(9)$ & $5.87(7)$ & $0.71(12)$ & $7.47(10)$ \\
\hline & & 5.3 & $5.17(6)$ & & $6.58(7)$ & $5.12(6)$ & & $6.52(7)$ \\
\hline & & 5.5 & $3.82(4)$ & & $4.86(4)$ & $3.78(4)$ & & $4.82(5)$ \\
\hline linear & 0.1 & 5.2 & $5.99(7)$ & $1.24(12)$ & $7.62(9)$ & $5.93(7)$ & $1.26(12)$ & $7.55(9)$ \\
\hline & & 5.3 & $5.21(6)$ & & $6.64(7)$ & $5.17(6)$ & & $6.58(7)$ \\
\hline & & 5.5 & $3.85(3)$ & & $4.91(4)$ & $3.82(4)$ & & $4.86(5)$ \\
\hline & ategy & & $10^{2} F_{\mathrm{K}}$ & $\alpha_{\mathrm{f}}$ & $10^{2} a[\mathrm{fm}]$ & $10^{2} F_{\mathrm{K}}$ & $\alpha_{\mathrm{f}}$ & $10^{2} a[\mathrm{fm}]$ \\
\hline$(5.15)$ & 0.15 & 5.2 & $5.86(7)$ & $1.30(6)$ & $7.45(9)$ & $5.77(7)$ & $1.42(6)$ & $7.35(9)$ \\
\hline & & 5.3 & $5.09(5)$ & & $6.48(7)$ & $5.01(5)$ & & $6.38(7)$ \\
\hline & & 5.5 & $3.76(3)$ & & $4.79(4)$ & $3.71(3)$ & & $4.72(4)$ \\
\hline & 0.1 & 5.2 & $5.88(7)$ & $1.13(8)$ & $7.49(9)$ & $5.83(7)$ & $1.15(8)$ & $7.42(9)$ \\
\hline & & 5.3 & $5.11(5)$ & & $6.51(7)$ & $5.06(6)$ & & $6.44(7)$ \\
\hline & & 5.5 & $3.79(3)$ & & $4.82(4)$ & $3.75(3)$ & & $4.77(4)$ \\
\hline linear & 0.1 & 5.2 & $5.97(7)$ & $1.78(8)$ & $7.61(9)$ & $5.92(7)$ & $1.72(8)$ & $7.54(9)$ \\
\hline & & 5.3 & $5.19(5)$ & & $6.61(7)$ & $5.15(5)$ & & $6.55(7)$ \\
\hline & & 5.5 & $3.84(3)$ & & $4.89(4)$ & $3.81(3)$ & & $4.85(4)$ \\
\hline
\end{tabular}

Table 5: Results for chiral fits in the range $y_{1} \leq y_{1}^{\max }$.

The fit parameters $\alpha_{4}$ and $\alpha_{\mathrm{f}}$ turn into low energy constants of $\mathrm{SU}(3)$ and $\mathrm{SU}(2)$ chiral perturbation theory, respectively, when the cuts on $y$ are sufficiently small, such that the used NLO expressions are accurate. A look in the tables shows that their dependence on the cut is anything but negligible. Clearly the values obtained with $y_{1}^{\max }=0.1$ are our best estimates. Differences to $y_{1}^{\max }=0.15$ are an estimate of the systematic error, which, however, deserves a more detailed study. We postpone this to a later analysis when data at an even smaller quark mass will be available for two values of the lattice spacing.

\section{Lambda parameter}

The $\Lambda$ parameter, which normalizes the scales in the perturbative running of QCD quantities at high energies, has been determined in an earlier work by the ALPHA collaboration [5] in terms of a length scale $L_{1}$. It used the Schrödinger functional which allows to compute the running over many orders of magnitude in the scale $L$, and $L_{1}$ is a typical hadronic scale which allows to make contact to physical 


\begin{tabular}{ccc}
\hline$\beta$ & $F_{\mathrm{K}}$ & $a[\mathrm{fm}]$ \\
\hline 5.2 & $0.0593(7)(6)$ & $0.0755(9)(7)$ \\
5.3 & $0.0517(6)(6)$ & $0.0658(7)(7)$ \\
5.5 & $0.0382(4)(3)$ & $0.0486(4)(5)$ \\
\hline
\end{tabular}

Table 6: The kaon decay constant at the physical quark mass and the corresponding lattice spacing. The first error is statistical, the second systematic error due to the chiral extrapolation.

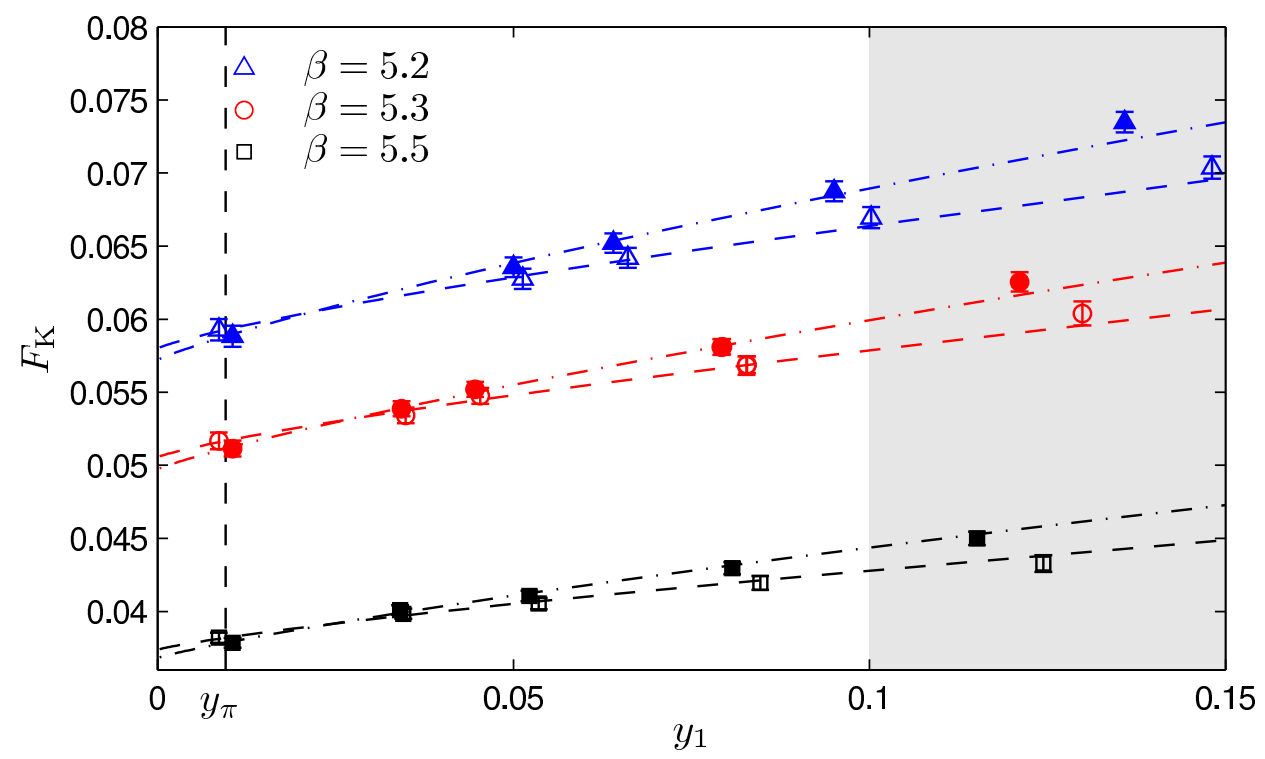

Figure 6: Chiral extrapolation of the kaon decay constant in lattice units for all three $\beta$. Open symbols and dashed lines correspond to strategy 1 , whereas filled symbols and dash-dotted lines represent strategy 2.

units.

In particular a renormalized coupling $\bar{g}(L)$ can be defined for which the renormalization group invariant $\Lambda$ parameter is given by

$$
L \Lambda=\left(b_{0} \bar{g}^{2}(L)\right)^{-b_{1} /\left(2 b_{0}^{2}\right)} e^{-1 /\left(2 b_{0} \bar{g}^{2}(L)\right)} \exp \left[-\int_{0}^{\bar{g}(L)} \mathrm{d} x\left\{\frac{1}{\beta(x)}+\frac{1}{b_{0} x^{3}}-\frac{b_{1}}{b_{0}^{2} x}\right\}\right]
$$

Here $\beta(\bar{g})=-L \partial \bar{g}(L) / \partial L$ with $b_{0}$ and $b_{1}$ the first two universal coefficients of its asymptotic expansion $\beta(\bar{g})=-\bar{g}^{3}\left(b_{0}+b_{1} \bar{g}^{2}+\ldots\right)$. Then $\Lambda$ is independent of the scale but dependent on the renormalization scheme adopted for the coupling. 


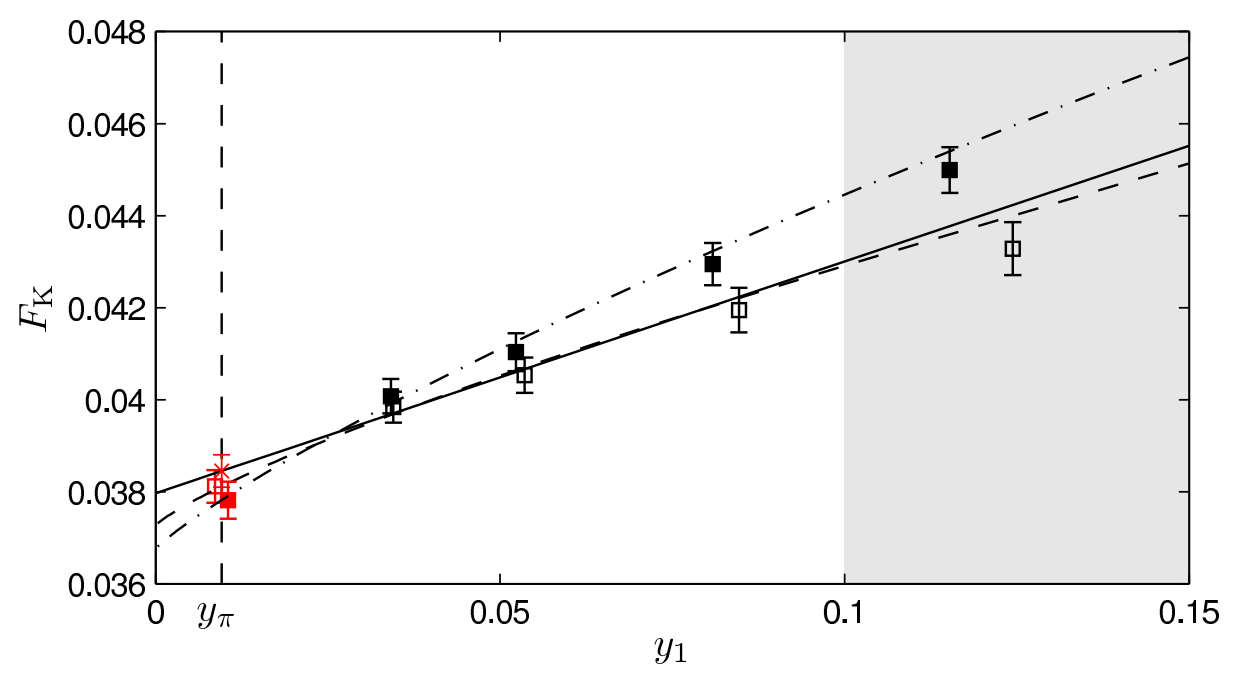

Figure 7: Detailed comparison of strategy 1, strategy 2 and a linear extrapolation at $\beta=5.5$. The symbols are the same as in Fig. 6 with the solid line representing the linear extrapolation of the open symbols. Note that the slopes given by the low energy parameters $\alpha$ are determined from the global fit to all three $\beta$.

The relation of the Schrödinger functional $\Lambda$ to its value in other schemes is given exactly by a one-loop perturbation theory computation.

For the scale at which we make contact to physical units we use $L_{1}$ defined through $\bar{g}^{2}\left(L_{1}\right)=4.484$. At this value of the coupling we have a large and precisely tuned set of pairs $\left(L_{1} / a, \beta\right)$. Reanalyzing the data of Ref. [5] at this point, we get the continuum value of $\Lambda L_{1}=0.264(15)$. What remains to be done is to compute $f_{\mathrm{K}} L_{1}$ in the continuum limit. To this end, we have to combine the updated data for $L_{1} / a$ of App. D with the decay constant $F_{\mathrm{K}}$ at physical quark masses described in the previous section. Since the data points for $L_{1} / a$ and $F_{\mathrm{K}}$ are at different values of $\beta$, we need to interpolate $L_{1} / a$ to the values of the latter; then the product $f_{\mathrm{K}} L_{1}$ can be extrapolated to the continuum limit.

\subsection{Interpolation of $L_{1}$}

We start with values of $L_{1} / a$ at $\beta=5.2,5.2638,5.4689,5.619,5.758,5.9631$ obtained in App. D and Ref. [47]. Since $L_{1}$ is a physical scale, we expect $\log \left(L_{1} / a\right)$ to be roughly linear in $\beta$. Four different fits have been tried: a linear fit to the full range in $\beta$ and to the range [5.2,5.619], which we compared to quadratic fits in the same intervals. The central values derived from these interpolations differ only by a small fraction of the assigned statistical uncertainty. Also the statistical errors of 


\begin{tabular}{ccccc}
\hline$\beta$ & $L_{1} / a$ & $L_{1} f_{\mathrm{K}}$ & $r_{0} / L_{1}$ & $\bar{m}_{\mathrm{s}} / f_{\mathrm{K}}$ \\
\hline 5.2 & $5.367(82)$ & $0.318(6)(3)$ & $1.155(22)$ & $0.530(12)(6)$ \\
5.3 & $6.195(51)$ & $0.320(5)(4)$ & $1.169(15)$ & $0.577(11)(7)$ \\
5.5 & $8.280(80)$ & $0.316(4)(2)$ & $1.213(17)$ & $0.617(11)(5)$ \\
\hline cont. & & $0.315(8)(2)$ & $1.252(33)$ & $0.678(12)(5)$ \\
\hline
\end{tabular}

Table 7: Values of $L_{1} / a, L_{1} f_{\mathrm{K}}, r_{0} / L_{1}$ and $\bar{m}_{\mathrm{s}} / f_{\mathrm{K}}$ at the three values of $\beta$. For the latter three, we also give the value extrapolated to the continuum limit. The running mass in the Schrödinger Functional scheme $\bar{m}_{\mathrm{s}}$ is given at the renormalization scale $L_{1}$. Statistical and systematic errors are given.

the interpolated points are virtually equal for these procedures, only the quadratic function to the restricted interval gives larger uncertainties, as expected from a fit with three parameters to four points. Since we want to obtain conservative estimates of the errors, we take the result of the latter in the further analysis. The three values of $L_{1} / a$ which we use in the following are listed in Table 7.

\subsection{Continuum value of the $\Lambda$ parameter}

Fig. 8 shows that in the continuum extrapolation of $L_{1} f_{\mathrm{K}}$ the cut-off effects are smaller than the statistical uncertainties; the numerical values are given in Table 7. Indeed, a constant extrapolation gives a $\chi^{2} /$ d.o.f. below unity. However, we use a linear extrapolation, allowing for $\mathrm{O}\left(a^{2}\right)$ effects hidden by the statistical fluctuations. ${ }^{8}$ In this extrapolation the covariance between the three points from the interpolation in $L_{1} / a$ is taken into account. For comparison with results in the literature, we give all results also in units of $\left.r_{0}\right|_{y_{1}=y_{\pi}}$. The two combinations evaluate to

$$
f_{\mathrm{K}} L_{1}=0.315(8)(2) \quad r_{0} / L_{1}=1.252(33)
$$

in the continuum limit.

As in the previous section, the final results come from strategy 1 for the chiral extrapolation of $F_{\mathrm{K}}$, given in Table 6 . Strategy 2 is used to estimate the systematic uncertainty; however, it is small compared to the statistical errors and

\footnotetext{
${ }^{8}$ That the leading corrections come at $\mathrm{O}\left(a^{2}\right)$ is based on the assumption that the two-loop approximation of the boundary improvement coefficient $c_{t}$ of the Schrödinger functional is sufficient at the accuracy we are aiming at. This is corroborated by experience from the quenched approximation [48] and earlier investigations of the approach to the continuum with $c_{t}$ at 1-loop and 2-loop precision [49].
} 


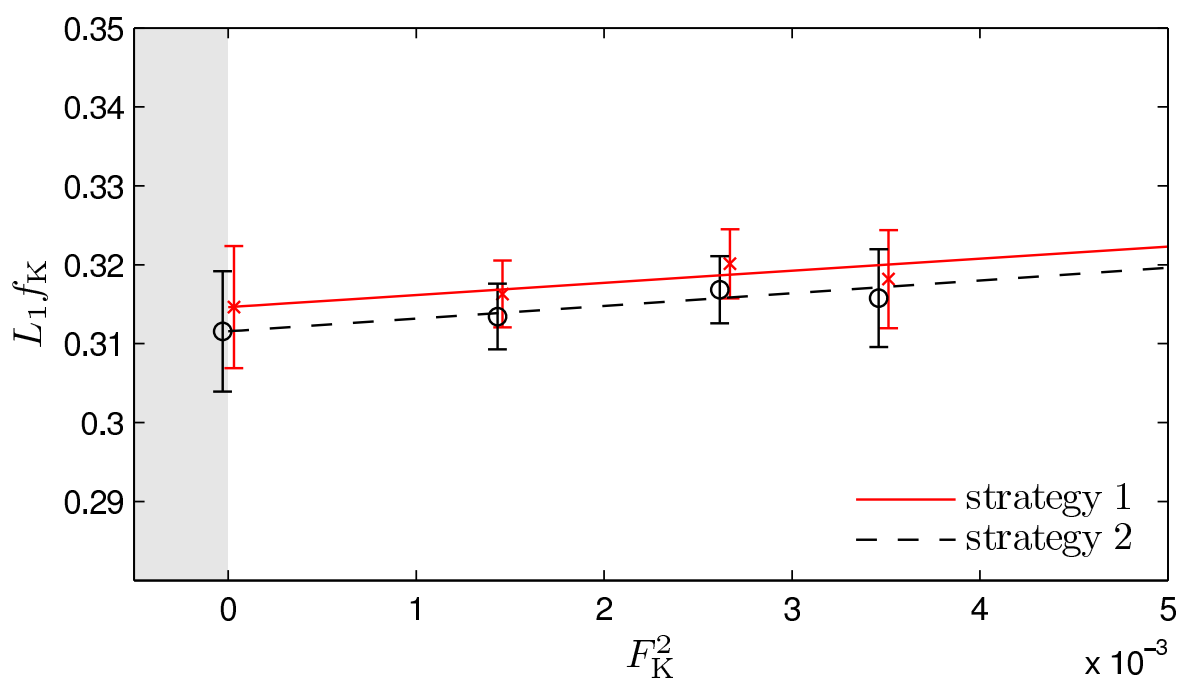

Figure 8: Continuum extrapolation of $L_{1}$ in units of $f_{\mathrm{K}}$. Even though the data shows no cutoff effects, we use a linear extrapolation to account for uncertainties from $\mathrm{O}\left(a^{2}\right)$ effects hidden by the errors. The two strategies for the chiral extrapolation of $F_{\mathrm{K}}=a f_{\mathrm{K}}$ agree well within statistics.

we do not give it separately. We therefore quote

$$
\Lambda^{(2)} / f_{\mathrm{K}}=0.84(6) \quad \text { and } \quad r_{0} \Lambda^{(2)}=0.331(22) .
$$

Now, as a result of our analysis, the error is dominated by the error on $\Lambda L_{1}$. We translate to the $\overline{\mathrm{MS}}$ scheme using $\Lambda_{\overline{\mathrm{MS}}}^{(2)}=2.382035(3) \Lambda^{(2)}[2,50]$ and find

$$
\Lambda \frac{(2)}{\mathrm{MS}}=310(20) \mathrm{MeV} \quad \text { and } \quad r_{0} \Lambda \frac{(2)}{\mathrm{MS}}=0.789(52) .
$$

\section{$7 \quad$ Strange quark mass}

The determination of the strange quark mass uses a strategy [51] which splits up the computation into several steps, circumventing the multi-scale problem a direct approach would face. It therefore allows for a good control over the systematic errors. Earlier results have been presented in Ref. [6], here we improve on them with a much higher statistical and systematic accuracy.

To briefly summarize the procedure, the RGI mass $^{9}$ and the renormalized running mass $\bar{m}_{\mathrm{s}}$ in the Schrödinger functional scheme are given in terms of the

\footnotetext{
${ }^{9}$ The RGI mass can be defined through $M_{\mathrm{s}}=\lim _{L \rightarrow 0}\left(2 b_{0} \bar{g}^{2}(L)\right)^{-d_{0} /\left(2 b_{0}\right)} \bar{m}_{\mathrm{s}}(L)$ with the universal coefficient $d_{0}=8 /(4 \pi)^{2}[52]$.
} 
bare PCAC mass $m_{\mathrm{s}}$ by

$$
M_{\mathrm{s}}=Z_{\mathrm{M}} m_{\mathrm{s}}=\frac{M}{\bar{m}(L)} \bar{m}_{\mathrm{s}}(L)=\frac{M}{\bar{m}(L)} \frac{Z_{\mathrm{A}}}{Z_{\mathrm{P}}(L)} m_{\mathrm{s}},
$$

where the computation of the renormalization constant $Z_{\mathrm{M}}$ is split in two steps. The continuum value of the universal first factor $M / \bar{m}$ has been computed in Ref. [6] for the two flavor theory which we consider here. It does not depend on the quark flavor or the lattice regularization. For the second factor, updated values of $Z_{\mathrm{P}}$ are presented in App. C. Of course, the first factor and $Z_{\mathrm{P}}$ have to be evaluated at a common renormalization scale $L$. While Ref. [6] used $L=L_{\max }$ with $\bar{g}^{2}\left(L_{\max }\right)=4.61$, we choose here $L=L_{1}$ with $\bar{g}^{2}\left(L_{1}\right)=4.484$ as in the previous section. The first factor in eq. (7.1) is $M / \bar{m}=1.308(16)$ at this scale from a re-evaluation of the data of Ref. [6].

\subsection{Determination of the strange quark mass}

For the bare strange quark mass at physical light quark masses $m_{\mathrm{s}}$, the final ingredient of eq. (7.1), we again follow the two strategies presented in Sec. 5. Of the two, the second holds the strange quark mass fixed as a function of the light quark mass and is therefore the natural choice for this analysis. We still use strategy 1 as a cross check to estimate systematic effects from the chiral extrapolation.

\subsubsection{Strange mass from strategy 2}

The hopping parameter of the strange quark $\kappa_{\mathrm{s}}$ is a non-trivial function of $\kappa_{1}$, defined in strategy 2 as

$$
\kappa_{\mathrm{s}}=s\left(\kappa_{1}, \mu_{\mathrm{s}}\right),
$$

with $s\left(\kappa_{1}, \mu\right)$ defined in eq. (5.14). Its numerical determination as well as the determination of $\mu_{\mathrm{s}}$ have been explained in Sect. 5.3. The resulting values of $\kappa_{\mathrm{s}}$ are listed in Table 1. This definition holds the bare PCAC strange quark mass fixed to the value $\mu_{\mathrm{s}}$ set by the physics requirement eq. (5.16) at physical light quarks.

Expressing $M_{\mathrm{s}}$ in units of $f_{\mathrm{K}}$ we eliminate $Z_{\mathrm{A}}$ and get

$$
\frac{M_{\mathrm{s}}}{f_{\mathrm{K}, \mathrm{phys}}}=\frac{M}{\bar{m}(L)} \times \frac{1}{Z_{\mathrm{P}}(L)}\left[1+\left(\tilde{b}_{\mathrm{A}}-\tilde{b}_{\mathrm{P}}\right) \mu_{\mathrm{s}}\right] \frac{\mu_{\mathrm{s}}}{F_{\mathrm{K}, \text { phys }}^{\text {bare }}},
$$

with $F_{\mathrm{K}, \text { phys }}^{\text {bare }}=F_{\mathrm{K} \text {,phys }} / Z_{\mathrm{A}}$. The second factor is $\mathrm{O}(a)$ improved, if we neglect, as before, a tiny correction $\left(\bar{b}_{\mathrm{A}}-\bar{b}_{\mathrm{P}}\right) a m_{\text {sea }}$. 


\subsubsection{Strategy 1}

Within this strategy, it is most natural to determine the combination $M_{\mathrm{s}}+\hat{M}$, the sum of strange and light quark mass, since this combination is kept fixed at lowest order in chiral perturbation theory along the trajectory defined by eq. (5.6). The first order corrections are easily incorporated from [45] such that we arrive after renormalization as in eq. (7.3) at

$$
\begin{aligned}
\frac{2 m_{13}\left(\kappa_{1}, h\left(\kappa_{1}\right)\right)}{Z_{\mathrm{P}} f_{\mathrm{K}}\left(\kappa_{1}, h\left(\kappa_{1}\right)\right)} & =\frac{\bar{m}_{\mathrm{s}}+\hat{m}}{f_{\mathrm{K}, \text { phys }}}\left[1+\bar{L}_{\mathrm{m}}\left(y_{1}, y_{\mathrm{K}}\right)+\left(\alpha_{4,6}-\frac{1}{4}\right)\left(y_{1}-y_{\pi}\right)+\mathrm{O}\left(y^{2}\right)\right] \\
\bar{L}_{\mathrm{m}}\left(y_{1}, y_{\mathrm{K}}\right) & =L_{\mathrm{m}}\left(y_{1}, y_{\mathrm{K}}\right)-L_{\mathrm{m}}\left(y_{\pi}, y_{\mathrm{K}}\right), \quad \alpha_{4,6}=3 \alpha_{4}-4 \alpha_{6} \\
L_{\mathrm{m}}\left(y_{1}, y_{\mathrm{K}}\right) & =-\left(y_{\mathrm{K}}-\frac{3}{8} y_{1}\right) \log \left(2 y_{\mathrm{K}} / y_{1}-1\right)-y_{\mathrm{K}} \log \left(y_{1}\right)
\end{aligned}
$$

which we can use to extract $\left(M_{\mathrm{s}}+\hat{M}\right) / f_{\mathrm{K} \text {,phys }}$ and the combination of low energy constants $\alpha_{4,6}$. Removing the contribution of the average light quark mass $\hat{M}$ amounts to multiplying by a correction factor such that $M_{\mathrm{s}}=\left(M_{\mathrm{s}}+\hat{M}\right)(1-\rho)$, where $\rho$ is a small number and can therefore be incorporated by its lowest order chiral perturbation theory estimate

$$
\rho \equiv \frac{\hat{M}}{M_{\mathrm{s}}+\hat{M}} \approx \frac{m_{\pi}^{2}}{2 m_{\mathrm{K}}^{2}}=0.037 .
$$

Because of this last approximation, we prefer the determination of $M_{\mathrm{s}}$ from strategy 2 and use the one here just as a consistency check.

\subsection{The strange quark mass in the continuum limit}

For strategy 2, the strange quark mass is the strange quark mass is directly kept fixed at its physical value. The discussion can be found in Sec. 5. For strategy 1, the extrapolation follows the same principles as before: we neglect cut-off effects in the NLO terms of the chiral expansion. The corresponding fit to the data is displayed in Fig. 9 on the left, which demonstrates that this assumption holds well within the statistical accuracy. Also shown for comparison is a linear extrapolation which agrees within the uncertainties with the values obtained from the ChPT formulae.

The values of $\bar{m}_{\mathrm{s}} / f_{\mathrm{K}}$ for the two strategies as a function of the lattice spacings are plotted in Fig. 9 on the right. Already at finite lattice spacing the two sets seem to be shifted with respect to another and therefore have parallel continuum extrapolations which, however, agree within uncertainties. The data fits well 

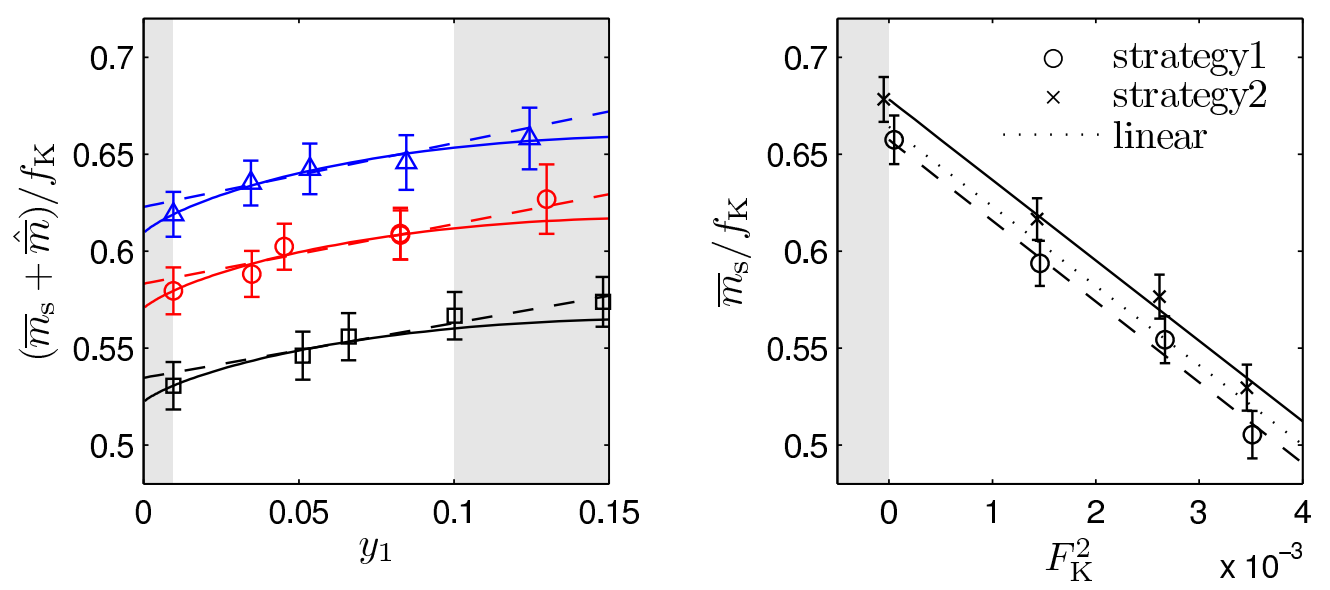

Figure 9: Left: Chiral extrapolation of the strange quark mass according to strategy 1 . The dashed line indicates a corresponding linear fit which gives a value that agrees within uncertainties at the physical point. Right: Continuum extrapolation of the strange quark mass in units of $f_{\mathrm{K}}$ for the results of the two strategies.

the assumption that only leading cut-off effects are present and we observe a correction of $8 \%$ from our value on the finest lattices. Since we prefer strategy 2 for conceptual reasons, we quote as a final result with statistical and systematic uncertainties

$$
\bar{m}_{\mathrm{s}} / f_{\mathrm{K}}=0.678(12)(5), \quad M_{\mathrm{s}} / f_{\mathrm{K}}=0.887(19)(7), \quad M_{\mathrm{s}}=138(3)(1) \mathrm{MeV},
$$

where we use $M / \bar{m}=1.308(16)$ as quoted before. For reference, we also give the numbers in the $\overline{\mathrm{MS}}$ scheme. This conversion is the only part of the computation in which we need to take recourse to perturbation theory, known in this case to four loops, which differs from the two- and three-loop result by only a small amount. We use the same method as described in Ref. [6], but with the new value of $\Lambda_{\overline{\mathrm{MS}}}$ which leads us to $\bar{m}^{\overline{\mathrm{MS}}}(2 \mathrm{GeV}) / M=0.740(12)$ and

$$
\bar{m}_{\mathrm{s}}^{\overline{\mathrm{MS}}}(2 \mathrm{GeV})=\frac{M_{\mathrm{s}}}{f_{\mathrm{K}}} \frac{\bar{m}^{\overline{\mathrm{MS}}}(2 \mathrm{GeV})}{M} f_{\mathrm{K}, \mathrm{phys}}=102(3)(1) \mathrm{MeV} .
$$

Here also the statistical uncertainty of $\Lambda_{\overline{\mathrm{MS}}}$ is included.

\section{Conclusions}

Setting the scale in lattice computations requires a dimensionful quantity which is known from experiment to good accuracy for which we chose the kaon decay constant. For currently available lattices this involves chiral extrapolations to the 
physical light quark masses, which can be important corrections depending on the quantity. Chiral perturbation theory turns out to be a good guide, if one stays within its domain of applicability. In particular the first strategy discussed in this paper, designed to have small chiral corrections for the kaon decay constant, has proven to be successful in the sense that the extrapolation is very robust under reasonable changes in the functional form: various next-to-leading order expansions and also a linear extrapolation give compatible results.

Whereas strategy 1 keeps the sum of strange and light quark mass approximately constant, strategy 2 provides a constant strange quark mass, making it suitable for the determination of $M_{\mathrm{s}}$ and further analysis of strange baryons. This flexibility is probably particular to the situation of a quenched strange quark, however, the fact that the results from both strategies agree within small uncertainties gives rise to the expectation that also with a dynamical strange quark and the $\mathrm{u} / \mathrm{d}$ quarks sufficiently in the chiral regime, the extrapolations can be safe.

As already mentioned in the introduction, this publication completes the $N_{\mathrm{f}}=2$ part of a long research program by the ALPHA collaboration, which has the determination of the $\Lambda$ parameter and the strange quark mass as two of its main goals. We can therefore quote these two observables for the two flavor theory in physical units

$$
\Lambda_{\overline{\mathrm{MS}}}^{(2)}=310(20) \mathrm{MeV}, \quad M_{\mathrm{s}}=138(3)(1) \mathrm{MeV},
$$

with the value of the strange quark mass in the $\overline{\mathrm{MS}}$ scheme given in eq. (7.9).

Since previous results in physical units were wrong due to inaccurate values for $r_{0} / a$ in the literature, we have also computed this scale and get

$$
r_{0}=0.503(10) \mathrm{fm}
$$

at the physical pion mass using $f_{\mathrm{K}}=155 \mathrm{MeV}$.

The restriction to two flavor QCD introduces some ambiguity to the scale setting and quark mass determination, because mass ratios do not have to be the same as in Nature. The effect of the heavier quarks is unknown and interesting in its own right. Comparing to the $N_{\mathrm{f}}=2$ results presented here, future calculations will shed light on the question of the contributions of strange and charm quarks in the sea.

For now we can make a comparison to the values obtained in the quenched approximation. In the strange quark mass we find no significant difference to the corresponding value quoted in Ref. [54]. However, the $\Lambda$ parameter exhibits an interesting dependence on $N_{\mathrm{f}}$, with the five flavor theory $\Lambda^{(5)}=212(12) \mathrm{MeV}$ extracted from Bethke's world average $\alpha_{\overline{\mathrm{MS}}}\left(M_{\mathrm{Z}}\right)=0.118(10)$ [53] and the quenched 


\begin{tabular}{cccc}
\hline$N_{\mathrm{f}}$ & $\Lambda_{\overline{\mathrm{MS}}}$ & experiment & theory \\
\hline 0 & $238(19) \mathrm{MeV}$ & $m_{\mathrm{K}}, K \rightarrow \mu \nu_{\mu}, K \rightarrow \pi \mu \nu_{\mu}$ & lattice gauge theory [3] \\
2 & $310(20) \mathrm{MeV}$ & $m_{\mathrm{K}}, K \rightarrow \mu \nu_{\mu}, K \rightarrow \pi \mu \nu_{\mu}$ & this work \\
\hline 5 & $212(12) \mathrm{MeV}$ & world average & perturb. theory [53] \\
\hline
\end{tabular}

Table 8: The $\Lambda$-parameter as a function of the number of flavors.

value $\Lambda^{(0)}=238(19) \mathrm{MeV}$ [3] listed in Table 8. Perturbation theory predicts $\Lambda^{\left(N_{\mathrm{f}}\right)}<\Lambda^{\left(N_{\mathrm{f}}-1\right)}$, which is valid and precise when the quark being decoupled is sufficiently heavy. This is expected to be the case for the b-quark and thus $\Lambda^{(5)}<\Lambda^{(4)}$. However, comparing the ALPHA value for $N_{\mathrm{f}}=0$ and our new $N_{\mathrm{f}}=2$ result shows that for light quarks the $N_{\mathrm{f}}$-dependence is in the opposite direction. The $\Lambda$ parameter increases quite strongly with increasing $N_{\mathrm{f}}$. An eventual decrease is likely to be present between $N_{\mathrm{f}}=3$ and $N_{\mathrm{f}}=4$, leading to an agreement with the phenomenological determinations of $\alpha_{\overline{\mathrm{MS}}}\left(M_{\mathrm{Z}}\right)$ [53].

In any case, it will be an important milestone to arrive at a non-perturbative and precise determination of $\Lambda^{(4)}$ and compare with the present world average. This is particularly needed in the light of the spread of different determinations before taking the world average. For example the authors of Ref. [55] analyse deep inelastic scattering data by a global fit to parton distribution functions and $\alpha_{\overline{\mathrm{MS}}}$ at NNLO. Their value for $\alpha_{\overline{\mathrm{MS}}}\left(M_{\mathrm{Z}}\right)$ corresponds to $\Lambda_{\overline{\mathrm{MS}}}^{(5)}=160(11) \mathrm{MeV}$, which is quite a bit smaller than the world average.

Acknowledgements. We acknowledge helpful discussions with O. Bär, M. Della Morte, M. Lüscher, H. Simma, U. Wolff and in general members of CLS. We are grateful for computer time allocated for our project on the Jugene and Juropa computers at $\mathrm{NIC}$, Jülich, and the ICE at $\mathrm{ZiB}$, Berlin. The lattice group at the University of Bielefeld kindly granted us access to their apeNEXT computers, which allowed us to improve the determination of the renormalization constants. This work is supported by the Deutsche Forschungsgemeinschaft in the SFB/TR 09 and the GRK 1504 "Masse, Spektrum, Symmetrie" as well as by the European community through EU Contract No. MRTN-CT-2006-035482, "FLAVIAnet". This work was granted access to the HPC resources of the Gauss Center for Supercomputing at Forschungzentrum Jülich, Germany, made available within the Distributed European Computing Initiative by the PRACE-2IP, receiving funding from the European Community's Seventh Framework Programme (FP7/2007-2013) under grant agreement RI-283493. 


\begin{tabular}{lcccccccc}
\hline id & block & $R_{\text {active }}$ & $\tau$ & $N_{0}$ & $N_{1}$ & $N_{2}$ & acc. rate & MD time \\
\hline A3 & $8^{4}$ & 0.37 & 2 & 4 & 5 & 125 & 0.91 & 8030 \\
A4 & $8^{4}$ & 0.37 & 2 & 4 & 5 & 150 & 0.85 & 8090 \\
\hline E5f & $8^{4}$ & 0.37 & 0.5 & 4 & 5 & 22 & 0.87 & 16000 \\
E5g & $8^{4}$ & 0.37 & 4 & 4 & 5 & 176 & 0.83 & 16180 \\
F6 & $8^{4}$ & 0.37 & 2 & 4 & 5 & 260 & 0.89 & 4800 \\
F7a & $8^{4}$ & 0.37 & 2 & 4 & 5 & 350 & 0.84 & 5600 \\
F7b & $8^{4}$ & 0.37 & 2 & 4 & 5 & 350 & 0.87 & 4000 \\
\hline N4 & $8^{2} 12^{2}$ & 0.44 & 0.5 & 4 & 5 & 24 & 0.88 & 3700 \\
N5 & $8^{2} 12^{2}$ & 0.44 & 0.5 & 4 & 5 & 24 & 0.87 & 3800 \\
\hline
\end{tabular}

Table 9: Parameters of the DD-HMC algorithm for the ensembles used in this publication. We give the HMC block size, the corresponding ratio of active links $R_{\text {active }}$, the trajectory length, the (relative) step sizes of the gauge, block fermion and global fermion force. This is followed by the measured acceptance rate and the total statistics after thermalization.

\section{A Details of the update algorithms}

In our simulation we first used the DD-HMC algorithm [14,15] as implemented in Lüscher's package [16] and then switched to our implementation of a mass preconditioned HMC [23]. Both algorithms have several parameters which influence their performance and we therefore give the essential ones in this section.

\section{A.1 DD-HMC}

The block decomposition of the DD-HMC algorithm separates the infrared from the ultraviolet modes. The details and notation can be found in Ref. [14]. We give the size of the blocks and the step sizes of the three different forces in Table 9. The gauge force has $N_{0} N_{1} N_{2}$ steps per trajectory, the block fermion force $N_{1} N_{2}$ and the global fermion force $N_{2}$ steps. The number of trajectories times their length is given in the "MD time" column of the table; to get an effective statistics, this can be multiplied by the ratio of active links $R_{\text {active }}$.

\section{A.2 MP-HMC}

In our implementation of the mass preconditioned HMC [23], we split the fermion determinant $\operatorname{det} Q^{2}$, with $Q=a \gamma_{5} D$, first by symmetric even-odd preconditioning

$$
\operatorname{det} Q^{2}=\operatorname{det}^{2} Q_{\mathrm{ee}} \operatorname{det}^{2} Q_{\mathrm{oo}} \operatorname{det}^{2} Q_{\mathrm{S}}
$$




\begin{tabular}{lccccccccc}
\hline id & $\kappa_{1}$ & $\kappa_{2}$ & $\tau$ & $N_{0}$ & $N_{1}$ & $N_{2}$ & $N_{3}$ & acc. rate & MD time \\
\hline A5c & 0.135887 & 0.134250 & 2 & 9 & 1 & 1 & 32 & 0.93 & 1160 \\
A5d & 0.135887 & 0.134250 & 2 & 9 & 1 & 1 & 32 & 0.92 & 1700 \\
\hline N6 & 0.136552 & 0.133857 & 2 & 9 & 1 & 1 & 16 & 0.84 & 4000 \\
O7 & 0.136550 & 0.135000 & 2 & 9 & 1 & 2 & 16 & 0.83 & 4000 \\
\hline
\end{tabular}

Table 10: Parameters of the MP-HMC algorithm for the ensembles used in this publication. The $\kappa_{i}$ parametrize the preconditioning masses and are followed by the trajectory length and the (relative) step numbers per trajectory. Also the acceptance rate and the statistics after thermalization are given.

and then apply two levels of mass preconditioning [21] to its Schur complement $Q_{\mathrm{S}}=1-Q_{\mathrm{ee}}^{-1} Q_{\mathrm{eo}} Q_{\mathrm{oo}}^{-1} Q_{\mathrm{oe}}$, i.e.,

$$
\operatorname{det} Q_{\mathrm{S}}^{2}=\operatorname{det}^{2}\left[W^{-1}\left(\sigma_{2}\right) W\left(\sigma_{1}\right)\right] \operatorname{det}^{2}\left[W^{-1}\left(\sigma_{1}\right) Q_{\mathrm{S}}\right] \operatorname{det}^{2}\left[W\left(\sigma_{2}\right)\right]
$$

with $W(\sigma)=Q_{\mathrm{S}}+\sigma$ and $\sigma_{2}>\sigma_{1}>0$. The forces from the individual contributions to the action are integrated on multiple time scales [56], the gauge force is on the finest level, the determinants of $Q_{\mathrm{ee}}$ and $Q_{\mathrm{oo}}$ are integrated together with the third determinant of eq. (A.2), the next two determinants constitute the two coarsest levels. For each we use a second order integrator [57], where an elementary step of size $\epsilon$ is given by

$$
T(\epsilon)=T_{1}(\lambda \epsilon) T_{2}(\epsilon / 2) T_{1}((1-2 \lambda) \epsilon) T_{2}(\epsilon / 2) T_{1}(\lambda \epsilon)
$$

$T_{1}$ is the force application on each level, whereas $T_{2}$ is again given by such a step with the force of the lower level. We use $\lambda=0.19$ for all levels. Only the gauge force is integrated with a standard leapfrog. Analogously to the DD-HMC, we give the relative step sizes in Table 10, note however, that eq. (A.3) implies a factor of two in the number of force applications between levels even for $N_{i}=1$. The preconditioning masses are given in terms of the hopping parameter such that $2 \sigma_{i}=\kappa_{i}^{-1}-\kappa_{\text {sea }}^{-1}$.

\section{B Improved determination of $Z_{\mathrm{A}}$}

The renormalization factor $Z_{\mathrm{A}}$ was previously computed [32] from a carefully chosen Ward identity. At $\beta=5.2$ an error of $2.1 \%$ resulted from the fact that at this $\beta$ an extrapolation in the quark mass was necessary. Since this uncertainty directly propagates into the strange quark mass determination and scale setting, 


\begin{tabular}{cccccc}
\hline$\beta$ & $L / a$ & $T / a$ & $\kappa$ & $Z_{\mathrm{A}}$ & $a m$ \\
\hline 5.20 & 12 & 18 & 0.13550 & $0.7853(15)$ & $0.02082(20)$ \\
& & & 0.13560 & $0.7792(20)$ & $0.01700(20)$ \\
& & & 0.13570 & $0.7767(20)$ & $0.01345(22)$ \\
& & & 0.13580 & $0.7757(27)$ & $0.00892(15)$ \\
\cline { 3 - 5 } & & & - & $0.771(6)$ & $\rightarrow 0$ \\
\hline
\end{tabular}

Table 11: Simulation parameters and results for $Z_{\mathrm{A}}$ and the PCAC mass $a m$ at $\beta=5.2$. The last row shows the chirally extrapolated value as described in the main text. To be compared with Table 1 of [32] and its chiral value $0.774(16)$.

we here improve $Z_{\mathrm{A}}$ at $\beta=5.2$. We substantially increased the statistics for the three previously simulated quark mass points and added another mass point, in order to better control the subsequent chiral extrapolation. We follow the lines of [32], i.e., in the determination of $Z_{\mathrm{A}}$ we use the optimal wave function of [29] to suppress the first excited state contribution in the pseudoscalar channel, use the "massive" definition of the renormalization constant [58], and we drop the disconnected quark diagrams which only amount to $\mathrm{O}\left(a^{2}\right)$ effects on $Z_{\mathrm{A}}$. In the notation of [32] we thus compute $Z_{\mathrm{A}}^{\text {con }}$ with $\omega=\omega_{\pi^{(0)}}$. The results are listed in Table 11.

We have performed several chiral extrapolations, including weighted averages of the two and three data points with the smallest masses, but in the end all resulting central values at $a m=0$ are covered by our preferred ansatz, a linear extrapolation of the data points with $a m<0.02$. It is shown in Fig. 10. The total range of the $y$-axis of the figure is the previous [32] $\pm 1 \sigma$ error. We now have a considerably improved value of $Z_{\mathrm{A}}=0.771(6)$. What remains to be done is to provide a global fit for $Z_{\mathrm{A}}\left(g_{0}^{2}\right)$. Again, several fit ansaetze have been tried and all of them give comparable results, especially in the $\beta$-range which is important in the present paper. Constraining the asymptotic behaviour to the perturbatively known one-loop estimate, we finally obtain

$$
\begin{aligned}
Z_{\mathrm{A}}\left(g_{0}^{2}\right) & =1-0.116458 g_{0}^{2}+c_{1} g_{0}^{4}+c_{2} g_{0}^{6}, \\
c & =\left(\begin{array}{l}
+1.16 \\
-7.21
\end{array}\right) \cdot 10^{-2}, \quad \operatorname{Cov}(c)=3 \cdot 10^{-4} \times\left(\begin{array}{cc}
+0.74 & -0.75 \\
-0.75 & +0.79
\end{array}\right),
\end{aligned}
$$

where $\operatorname{Cov}(c) / 3$ is the covariance matrix of the parameters $c_{1}, c_{2}$ obtained directly from the statistical errors of the data points. Due to the constraint fit ansatz with just two parameters, the error of the fit is significantly smaller than that of the individual data points. We therefore recommend to conservatively use $\operatorname{Cov}(c)$ 


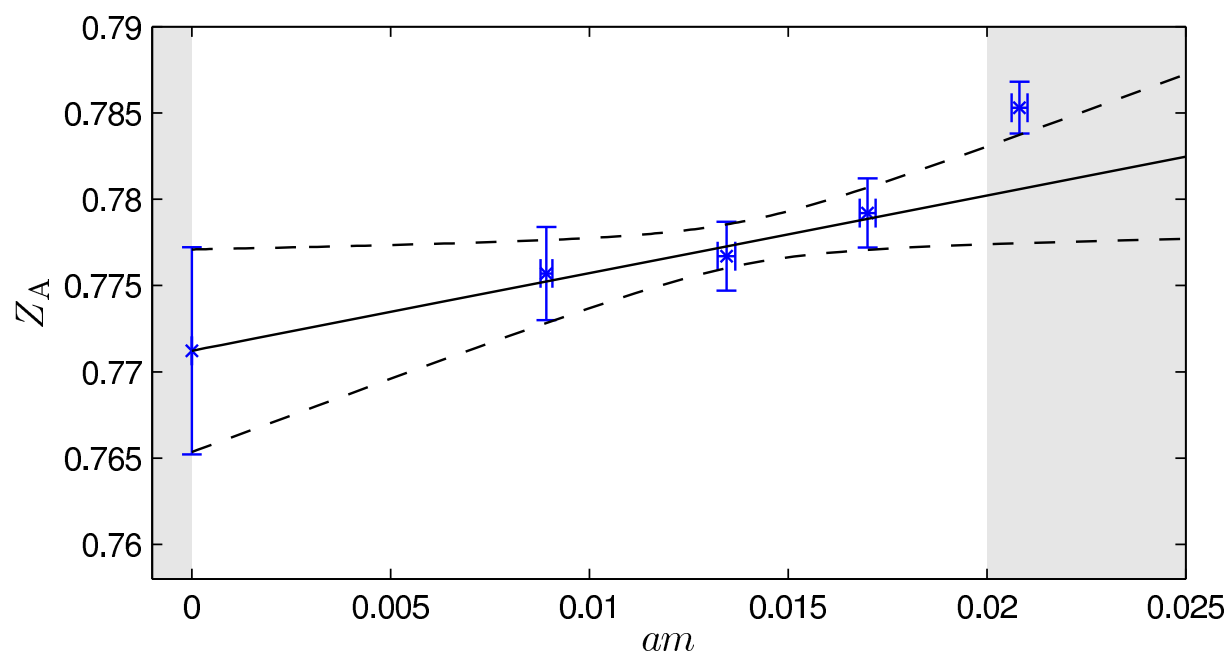

Figure 10: Data points with highly improved statistics for $Z_{\mathrm{A}}$ at $\beta=5.2$. The point in the chiral limit is obtained by a linear extrapolation of the three lightest PCAC masses.

with the factor 3 applied as above, which yields the grey error band in Fig. 11.

\section{Determination of $Z_{\mathrm{P}}$}

From the simulations reported in [47] we also have values for $Z_{\mathrm{P}}$, the renormalization constant of the pseudoscalar density. They are based on simulations with 8000 essentially uncorrelated measurements and thus have a very small statistical uncertainty. However, the small mismatch of the simulated $(L / a, \beta, a m)$ has to be accounted for and introduces additional errors. We need to distinguish $\beta=5.2$ and $\beta \geq 5.2638$, since in the latter case, we only have very small shifts.

We start with $\beta=5.2$. Here we have two different sea quark masses each at $L / a=4,6$ (see also Table 13). They enclose $L m=0$. We thus first perform an interpolation to vanishing mass and subsequently interpolate $\ln Z_{\mathrm{P}}$ to our target coupling linearly in $\ln \bar{g}^{2}$, c.f. Ref. [6]. A systematic error is estimated by comparing to an interpolation linear in $\bar{g}^{2}$.

Now we turn to $\beta \geq 5.2638$. Both the shifts to $a m=0, \bar{g}^{2}=4.484$ and the propagation of the errors in $\bar{g}^{2}(L)$ of Table 13 is done by a first order expansion of

$$
F(x, y)=\left.\ln Z_{\mathrm{P}}\right|_{\beta=\text { const }} \quad \text { with } x=\ln \left(\bar{g}^{2}\right), y=L m,
$$

in $x, y$. The derivative $\partial_{x} F$ is computed from perturbation theory, using the known (continuum) two-loop and three-loop perturbative results for $\tau(\bar{g})$ and 


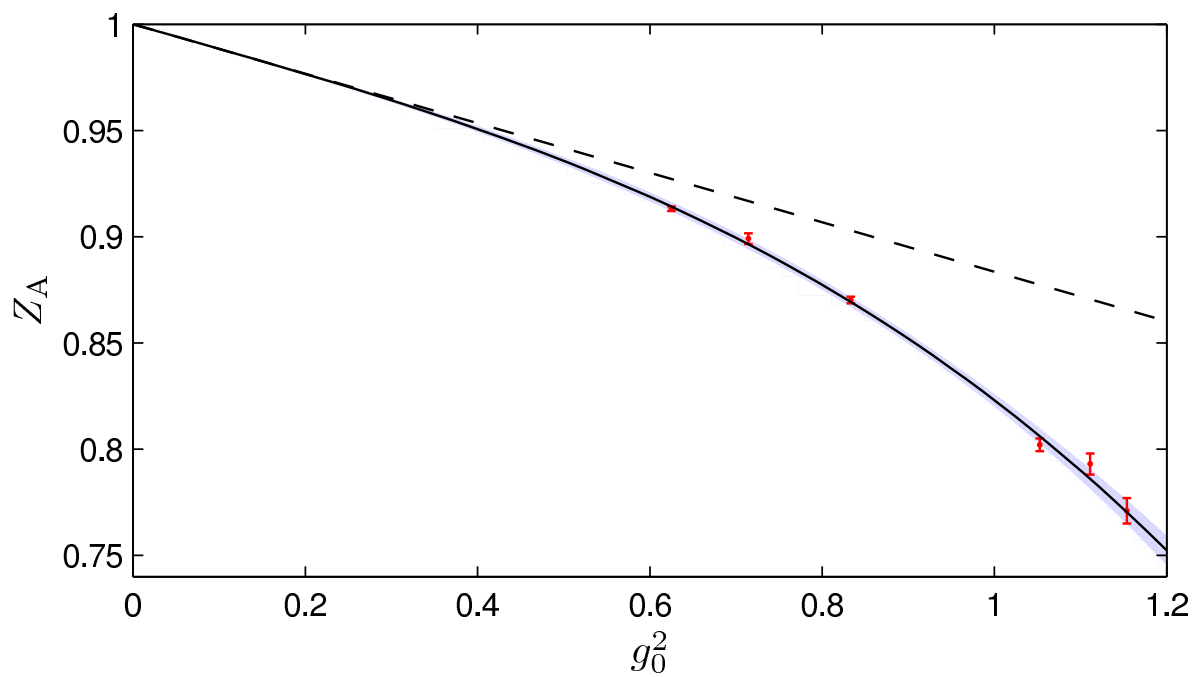

Figure 11: Result for our global fit of $Z_{\mathrm{A}}\left(g_{0}^{2}\right)$, together with the error band from eq. (B.1). For comparison we also show the perturbative (1-loop) result for $N_{\mathrm{f}}=2$.

$\beta(\bar{g})$, respectively. Note that perturbation theory is known to be rather accurate for the running of the mass (and therefore $Z_{\mathrm{P}}$ ) in the SF-scheme [6]. The other derivative is estimated as follows. At $\beta=5.2, L / a=4,6$ we have two mass points each and estimate $\partial_{y} F$ for $\bar{g}^{2}=3.80,4.95$ by finite differences. These are then interpolated to $\partial_{y} F(\ln (4.484), 0)=0.148(30)$. Up to tiny effects coming from the renormalization of $m$, the derivative $\partial_{y} F$ is universal. We neglect the $a^{2} / L^{2}$ corrections to exact universality, using $\partial_{y} F(\ln (4.484), 0)=0.148(30)$ at all $\beta$.

The results for $Z_{\mathrm{P}}$ are collected in Table 12. Apart from the special case $\beta=5.2$, where the interpolation in $\bar{g}^{2}$ introduces a noticeable uncertainty, all errors in the last column are dominated by the statistical errors in $\bar{g}^{2}\left(L_{1}\right)$.

Within our uncertainties the $g_{0}^{2}$-dependence of $Z_{\mathrm{P}}$ at fixed $L=L_{1}$ is not clearly visible. We thus take the average value of all six results and assign an error of about $1 \%$ to cover all results:

$$
Z_{\mathrm{P}}=0.5184(53), \quad 5.2 \leq \beta \leq 6.0 .
$$

\section{Improved determination of $L_{1}$}

The practical reason for $\bar{g}^{2}\left(L_{1}\right)=4.484$ instead of $\bar{g}^{2}\left(L_{\max }\right)=4.61$, chosen in [5], is that meanwhile dedicated tuning runs were carried out [47], which fix the 


\begin{tabular}{cccccc}
\hline$L / a$ & $\beta$ & $\kappa$ & $a m$ & $Z_{\mathrm{P}}(L, \beta, a m)$ & $Z_{\mathrm{P}}\left(L_{1}, \beta, 0\right)$ \\
\hline 4 & 5.2 & 0.135041 & $-0.00279(14)$ & $0.57672(15)$ & - \\
& & 0.134800 & $+0.00464(20)$ & $0.58291(22)$ & - \\
& & & $\rightarrow 0$ & $0.57904(13)$ & - \\
6 & 5.2 & 0.135617 & $-0.00747(20)$ & $0.48796(28)$ & - \\
& & 0.135226 & $+0.00571(17)$ & $0.49641(24)$ & - \\
& & & $\rightarrow 0$ & $0.49275(18)$ & - \\
\hline & 5.2 & & 0 & - & $0.5237(45)$ \\
6 & 5.2638 & 0.135985 & $-0.00585(18)$ & $0.50676(28)$ & $0.5150(16)$ \\
8 & 5.4689 & 0.136700 & $-0.00339(13)$ & $0.51103(29)$ & $0.5170(17)$ \\
10 & 5.6190 & 0.136785 & $-0.00260(9)$ & $0.51255(30)$ & $0.5186(25)$ \\
12 & 5.7580 & 0.136623 & $+0.00040(6)$ & $0.51856(29)$ & $0.5187(43)$ \\
16 & 5.9631 & 0.136422 & $-0.00107(4)$ & $0.51879(35)$ & $0.5172(48)$ \\
\hline
\end{tabular}

Table 12: Pseudoscalar renormalization constant $Z_{\mathrm{P}}$ from simulations in the Schrödinger functional. The kinematical setup is different from those in Table 13 and thus the PCAC mass am differs. The rightmost column lists the results after correcting for an imperfect tuning as explained in the text. For $\beta=5.2$, two non-zero quark masses have been simulated, allowing for an interpolation to vanishing mass.

simulation parameters $\left(L / a, \beta, \kappa_{\text {sea }}\right)$ at the line of constant physics

$$
\bar{g}^{2}\left(L_{1}\right)=4.484, \quad L_{1} m=0 .
$$

Here $m$ is the PCAC mass of the degenerate sea quarks and additional details can be found in the appendices of [47]. Of course fixing such a line of constant physics is only possible within a certain (statistical) uncertainty. While in the results of [47] the error due to the line of constant physics is not so relevant, we here aim for a more precise setting in order to extract the $\Lambda$ parameter and the scale $L_{1}$ in physical units. For this reason we have set up new simulations for $\bar{g}^{2}$ at $\beta \in\{5.2,5.2638,5.4689,5.6190\}$, corresponding to the first five sets of parameters in Table 13 which enclose the $\beta$-values used here. For completeness we also list the additional two sets for $\beta \in\{5.7580,5.9631\}$, taken over from the aforementioned reference. The simulations for $\bar{g}^{2}$ proceed along the lines of [5]. Results are listed in Table 13 together with the deviation $\delta\left[\bar{g}^{2}\right]=\bar{g}^{2}(L)-4.484$. At $\beta=5.2$ there is a large deviation since our code does not allow to simulate $L / a=5$; we have to rely on neighbouring points with $L / a=4,6$. At each of these values of $L / a$ we have simulations at slightly positive and negative $m$ from which we interpolate to $L m=0$, see Table 13 . We finally just take the value from $L / a=6$ into account, since it is closer to our target $\bar{g}^{2}$. At fixed lattice spacing we now take the results 


\begin{tabular}{ccccccc}
\hline$L / a$ & $\beta$ & $\kappa_{\text {sea }}$ & $a m$ & $\bar{g}^{2}(L)$ & $\delta\left[\bar{g}^{2}\right]$ & $L_{1} / a$ \\
\hline 4 & 5.2000 & 0.134700 & $-0.03745(41)$ & $3.730(11)$ & & \\
4 & 5.2000 & 0.133780 & $-0.00086(35)$ & $3.797(11)$ & & \\
4 & 5.2000 & - & $\rightarrow 0$ & $3.798(11)$ & -0.686 & $5.11(3)(13)$ \\
6 & 5.2000 & 0.135600 & $-0.01322(26)$ & $4.810(32)$ & & \\
6 & 5.2000 & 0.135200 & $+0.00289(24)$ & $4.984(33)$ & & \\
6 & 5.2000 & - & $\rightarrow 0$ & $4.954(33)$ & +0.470 & $5.33(4)(11)$ \\
\hline 6 & 5.2638 & 0.135673 & $+0.00012(19)$ & $4.550(25)$ & +0.066 & $5.89(4)(2)$ \\
8 & 5.4689 & 0.136575 & $+0.00046(11)$ & $4.526(32)$ & +0.042 & $7.91(7)(1)$ \\
10 & 5.6190 & 0.136700 & $+0.00038(8)$ & $4.531(51)$ & +0.037 & $9.87(14)(2)$ \\
\hline \hline 12 & 5.7580 & 0.136623 & $+0.00067(7)$ & $4.501(91)$ & +0.017 & $11.94(31)(1)$ \\
16 & 5.9631 & 0.136422 & $-0.00096(4)$ & $4.40(10)$ & +0.084 & $16.40(50)(6)$ \\
\hline
\end{tabular}

Table 13: Values of $L_{1} / a$ after correcting the simulated values $L / a$. For completeness we also include the two largest $\beta$-values, where no new simulations enter. The second error on the final result is the systematic one.

of $\bar{g}^{2}(L)$ as computed at integer $L / a$ and correct for the desired value $L_{1} / a$ at $\bar{g}^{2}\left(L_{1}\right)=4.484$, using

$$
\ln \left[\frac{L_{1}}{a}\right]=\ln \left[\frac{L}{a}\right]+\int_{\bar{g}\left(L_{1}\right)}^{\bar{g}(L)} \frac{\mathrm{d} x}{\beta(x)} .
$$

The non-perturbative estimate of the $\beta$-function from [5] is inserted, but we treat the difference to the value of $L_{1} / a$, obtained from perturbation theory at the highest available order, as a systematic uncertainty. ${ }^{10}$ We quote this as second error in our results for $L_{1} / a$ in Table 13, the first error is due to the statistical error of $\bar{g}^{2}(L)$. Our final numbers to be used in Section 6.1 are those shown in the last column of Table 13 .

\section{E Critical mass $m_{\mathrm{cr}}$}

Since Wilson fermions explicitly break chiral symmetry, the quark masses experience an additive renormalization $m_{\mathrm{cr}}$. It is defined by the point where the PCAC quark mass eq. (3.3) vanishes for quarks degenerate to the sea quarks: $m_{12}=0$. Even though we have chosen our improvement procedure in terms of $m_{r s}$ and hence do not need $m_{\mathrm{cr}}$ in our analysis, we give here its determination for completeness.

\footnotetext{
${ }^{10}$ The estimate of $\beta(x)$ in [5] applies to the continuum step scaling function, while we here apply it for not-so-large $L / a$. This represents a second reason to prefer $L / a=6$ to $L / a=4$ in our determination at $\beta=5.2$.
} 


\begin{tabular}{cccc}
\hline$\beta$ & $\kappa_{\mathrm{cr}}$ & $Z r_{\mathrm{m}}$ & $r_{\mathrm{m}}$ \\
\hline 5.2 & $0.1360546(25)(30)$ & $1.438(20)(30)$ & $1.549(42)$ \\
5.3 & $0.1364572(11)(30)$ & $1.310(11)(20)$ & $1.323(23)$ \\
5.5 & $0.1367749(06)(04)$ & $1.228(06)(05)$ & $1.157(08)$ \\
\hline
\end{tabular}

Table 14: Value of the critical hopping parameter and the renormalization constant $Z r_{\mathrm{m}}$ and $r_{\mathrm{m}}$ for our three values of the coupling constant. The first error in $Z r_{\mathrm{m}}$ is statistical, the second systematic from a variation of the functional form and the range of the fit to eq. (E.1). For $r_{\mathrm{m}}$ these errors are added in quadrature together with the one from $Z$.

Up to terms of order $a^{2}$ the subtracted bare quark mass $m_{\mathrm{q}}=m_{0}-m_{\mathrm{cr}}$ is proportional to the quark mass from the plateau of eq. (3.3)

$$
m_{12}\left(1+\tilde{b}_{\mathrm{r}} a m_{12}\right)=Z_{\mathrm{m}} \frac{Z_{\mathrm{P}}}{Z_{\mathrm{A}}} r_{\mathrm{m}} m_{\mathrm{q}} \equiv Z r_{\mathrm{m}} m_{\mathrm{q}}
$$

which we use to define the subtraction term $m_{\mathrm{cr}}$. The improvement coefficient $\tilde{b}_{\mathrm{r}}$ is a linear combination of those improving the axial current, the pseudoscalar density and the bare subtracted quark mass while $Z_{\mathrm{m}}$ renormalizes quark mass differences $m_{\mathrm{q}, i}-m_{\mathrm{q}, j}$ and $Z_{\mathrm{m}} r_{\mathrm{m}}$ renormalizes the trace of the bare subtracted mass matrix $\sum_{i} m_{\mathrm{q}, i}[30]$. In one-loop perturbation theory, $\tilde{b}_{\mathrm{r}}=\frac{1}{2}+0.0500 g_{0}^{2}$ [31,59], which we expect to be a sufficient approximation since our quark masses are small. Results are given in Table 14, where we quote $\kappa_{\mathrm{cr}}=\left(2 a m_{\mathrm{cr}}+8\right)^{-1}$ instead of $m_{\mathrm{cr}}$ and use the non-perturbative $Z=1+0.090514 g_{0}^{2}\left(1-0.3922 g_{0}^{4}-0.2145 g_{0}^{6}\right) /\left(1-0.6186 g_{0}^{4}\right)$ of [46] to determine $r_{\mathrm{m}}$ from $Z r_{\mathrm{m}}$. The central values are from fits of eq. (E.1) to the data with $a m_{12}<0.01$; the resulting $\chi^{2} /$ d.o.f. are all smaller than 1 , which might be an indication that the errors on $m$ are overestimated. The systematic error is estimated from including also a free cubic term on the right hand side of eq. (E.1) and a variation of the fit range up to $a m_{12}=0.016$.

These non-perturbative values for $Z r_{\mathrm{m}}$ and $r_{\mathrm{m}}$ deviate significantly from the one-loop expectation $Z r_{\mathrm{m}}=1+0.090514 g_{0}^{2}$ [59], which is roughly $Z r_{\mathrm{m}} \simeq 1.1$ in our region of couplings and even more so for $r_{\mathrm{m}}$ which is one up to $\mathrm{O}\left(g_{0}^{4}\right)$ corrections.

\section{F Masses of the pseudoscalar mesons, decay constants and quark masses}

Following the analysis described in Sect. 3.2, we determined starting time slices $x_{0}^{\min } / a$ whose value for the ensembles we give in Table 15 . We give one value each 
for the mesons composed of two sea quarks, a sea and a strange quark and two strange quarks. We list the raw data of quark masses, pseudoscalar masses and decay constants in Tables 16-18.

\begin{tabular}{lcccccccccccc}
\hline & A2 & A3 & A4 & A5 & E4 & E5 & F6 & F7 & N4 & N5 & N6 & O7 \\
\hline$\left(\kappa_{1}, \kappa_{2}\right)$ & 17 & 17 & 17 & 13 & 15 & 20 & 17 & 17 & 26 & 23 & 23 & 23 \\
$\left(\kappa_{1}, \kappa_{3}\right)$ & 18 & 18 & 18 & 15 & 16 & 22 & 22 & 21 & 27 & 27 & 28 & 28 \\
$\left(\kappa_{3}, \kappa_{4}\right)$ & 19 & 19 & 19 & 19 & 18 & 24 & 24 & 26 & 23 & 29 & 31 & 31 \\
\hline
\end{tabular}

Table 15: Values of $x_{0}^{\min } / a$ used in the analysis, from top to bottom, for mesons made of two sea quarks, a sea and a strange quark and two strange quarks. 


\begin{tabular}{|c|c|c|c|c|c|}
\hline & $\kappa_{r}$ & $\kappa_{s}$ & $a m_{r s}$ & $a m_{\mathrm{PS}}$ & $F_{\mathrm{PS}}^{\text {bare }}$ \\
\hline \multirow{8}{*}{$\mathrm{A} 2$} & 0.13510 & 0.13510 & $0.02934(5)$ & $0.3250(5)$ & $0.1009(2)$ \\
\hline & 0.13530 & 0.13530 & $0.02438(5)$ & $0.2968(5)$ & $0.0977(2)$ \\
\hline & 0.13550 & 0.13550 & $0.01946(5)$ & $0.2661(5)$ & $0.0943(2)$ \\
\hline & 0.13570 & 0.13570 & $0.01454(5)$ & $0.2315(6)$ & $0.0908(2)$ \\
\hline & 0.13565 & 0.13510 & $0.02252(5)$ & $0.2858(5)$ & $0.0963(2)$ \\
\hline & & 0.13530 & $0.02007(5)$ & $0.2702(5)$ & $0.0947(2)$ \\
\hline & & 0.13550 & $0.01762(5)$ & $0.2537(6)$ & $0.0930(2)$ \\
\hline & & 0.13565 & $0.01578(5)$ & $0.2407(6)$ & $0.0917(2)$ \\
\hline \multirow{9}{*}{ A3 } & 0.13510 & 0.13510 & $0.02707(5)$ & $0.3078(4)$ & $0.0965(2)$ \\
\hline & 0.13530 & 0.13530 & $0.02211(5)$ & $0.2785(5)$ & $0.0930(2)$ \\
\hline & 0.13550 & 0.13550 & $0.01720(5)$ & $0.2464(5)$ & $0.0893(2)$ \\
\hline & 0.13570 & 0.13570 & $0.01231(6)$ & $0.2096(6)$ & $0.0854(2)$ \\
\hline & 0.13580 & 0.13510 & $0.01842(5)$ & $0.2551(5)$ & $0.0899(2)$ \\
\hline & & 0.13530 & $0.01597(5)$ & $0.2379(6)$ & $0.0882(2)$ \\
\hline & & 0.13550 & $0.01353(5)$ & $0.2194(6)$ & $0.0863(2)$ \\
\hline & & 0.13570 & $0.01109(5)$ & $0.1993(7)$ & $0.0843(2)$ \\
\hline & & 0.13580 & $0.00985(5)$ & $0.1883(6)$ & $0.0832(2)$ \\
\hline \multirow{9}{*}{$\mathrm{A} 4$} & 0.13510 & 0.13510 & $0.02557(5)$ & $0.2958(5)$ & $0.0933(2)$ \\
\hline & 0.13530 & 0.13530 & $0.02061(5)$ & $0.2659(5)$ & $0.0896(2)$ \\
\hline & 0.13550 & 0.13550 & $0.01571(5)$ & $0.2328(5)$ & $0.0857(2)$ \\
\hline & 0.13570 & 0.13570 & $0.01087(5)$ & $0.1948(7)$ & $0.0814(2)$ \\
\hline & 0.13590 & 0.13510 & $0.01574(5)$ & $0.2334(6)$ & $0.0851(2)$ \\
\hline & & 0.13530 & $0.01330(5)$ & $0.2150(6)$ & $0.0833(2)$ \\
\hline & & 0.13550 & $0.01087(5)$ & $0.1949(7)$ & $0.0813(2)$ \\
\hline & & 0.13570 & $0.00845(5)$ & $0.1726(7)$ & $0.0791(2)$ \\
\hline & & 0.13590 & $0.00601(6)$ & $0.1466(8)$ & $0.0766(3)$ \\
\hline \multirow{9}{*}{ A5 } & 0.13510 & 0.13510 & $0.02488(5)$ & $0.2907(6)$ & $0.0918(2)$ \\
\hline & 0.13530 & 0.13530 & $0.01993(5)$ & $0.2605(6)$ & $0.0881(2)$ \\
\hline & 0.13550 & 0.13550 & $0.01504(5)$ & $0.2269(6)$ & $0.0841(2)$ \\
\hline & 0.13570 & 0.13570 & $0.01021(5)$ & $0.1882(7)$ & $0.0797(2)$ \\
\hline & 0.13594 & 0.13510 & $0.01460(5)$ & $0.2244(6)$ & $0.0828(2)$ \\
\hline & & 0.13530 & $0.01217(5)$ & $0.2053(7)$ & $0.0810(2)$ \\
\hline & & 0.13550 & $0.00975(5)$ & $0.1844(7)$ & $0.0789(2)$ \\
\hline & & 0.13570 & $0.00734(5)$ & $0.1608(8)$ & $0.0766(3)$ \\
\hline & & 0.13594 & $0.00444(5)$ & $0.1263(8)$ & $0.0735(3)$ \\
\hline
\end{tabular}

Table 16: Partially quenched average quark masses, pseudoscalar meson masses and decay constants in lattice units for $\beta=5.2$. 


\begin{tabular}{|c|c|c|c|c|c|}
\hline & $\kappa_{r}$ & $\kappa_{s}$ & $a m_{r s}$ & $a m_{\mathrm{PS}}$ & $F_{\mathrm{PS}}^{\mathrm{bare}}$ \\
\hline \multirow{9}{*}{ E4 } & 0.13540 & 0.13540 & $0.03121(7)$ & $0.3018(12)$ & $0.0884(5)$ \\
\hline & 0.13560 & 0.13560 & $0.02589(8)$ & $0.2746(13)$ & $0.0852(5)$ \\
\hline & 0.13580 & 0.13580 & $0.02061(8)$ & $0.2449(13)$ & $0.0819(5)$ \\
\hline & 0.13600 & 0.13600 & $0.01535(8)$ & $0.2118(14)$ & $0.0784(5)$ \\
\hline & 0.13610 & 0.13540 & $0.02191(8)$ & $0.2529(14)$ & $0.0825(5)$ \\
\hline & & 0.13560 & $0.01928(8)$ & $0.2372(14)$ & $0.0809(5)$ \\
\hline & & 0.13580 & $0.01666(8)$ & $0.2206(14)$ & $0.0793(5)$ \\
\hline & & 0.13600 & $0.01404(9)$ & $0.2028(15)$ & $0.0775(5)$ \\
\hline & & 0.13610 & $0.01272(9)$ & $0.1934(15)$ & $0.0767(6)$ \\
\hline \multirow{9}{*}{ E5 } & 0.13540 & 0.13540 & $0.02968(3)$ & $0.2896(3)$ & $0.08509(17)$ \\
\hline & 0.13560 & 0.13560 & $0.02436(3)$ & $0.2619(3)$ & $0.08173(17)$ \\
\hline & 0.13580 & 0.13580 & $0.01907(3)$ & $0.2318(3)$ & $0.07809(17)$ \\
\hline & 0.13600 & 0.13600 & $0.01383(3)$ & $0.1979(4)$ & $0.07413(17)$ \\
\hline & 0.13625 & 0.13540 & $0.01840(3)$ & $0.2281(4)$ & $0.07672(17)$ \\
\hline & & 0.13560 & $0.01578(3)$ & $0.2114(4)$ & $0.07514(17)$ \\
\hline & & 0.13580 & $0.01317(3)$ & $0.1934(4)$ & $0.07337(17)$ \\
\hline & & 0.13600 & $0.01056(3)$ & $0.1737(4)$ & $0.07139(17)$ \\
\hline & & 0.13625 & $0.00727(3)$ & $0.1454(4)$ & $0.06852(20)$ \\
\hline \multirow{9}{*}{ F6 } & 0.13540 & 0.13540 & $0.02874(2)$ & $0.2824(3)$ & $0.08294(18)$ \\
\hline & 0.13560 & 0.13560 & $0.02341(2)$ & $0.2542(3)$ & $0.07947(18)$ \\
\hline & 0.13580 & 0.13580 & $0.01812(3)$ & $0.2234(3)$ & $0.07570(19)$ \\
\hline & 0.13600 & 0.13600 & $0.01288(3)$ & $0.1885(3)$ & $0.0716(2)$ \\
\hline & 0.13635 & 0.13540 & $0.01616(3)$ & $0.2113(4)$ & $0.0729(2)$ \\
\hline & & 0.13560 & $0.01354(3)$ & $0.1935(4)$ & $0.0713(2)$ \\
\hline & & 0.13580 & $0.01092(3)$ & $0.1741(4)$ & $0.0695(2)$ \\
\hline & & 0.13600 & $0.00832(3)$ & $0.1524(4)$ & $0.0674(2)$ \\
\hline & & 0.13635 & $0.00374(3)$ & $0.1036(5)$ & $0.0629(3)$ \\
\hline \multirow{9}{*}{ F7 } & 0.13540 & 0.13540 & $0.028455(17)$ & $0.2801(2)$ & $0.08208(14)$ \\
\hline & 0.13560 & 0.13560 & $0.023121(19)$ & $0.2520(3)$ & $0.07857(14)$ \\
\hline & 0.13580 & 0.13580 & $0.017832(19)$ & $0.2211(3)$ & $0.07473(15)$ \\
\hline & 0.13600 & 0.13600 & $0.012594(20)$ & $0.1860(3)$ & $0.07045(16)$ \\
\hline & 0.13638 & 0.13540 & $0.01549(2)$ & $0.2068(3)$ & $0.07122(18)$ \\
\hline & & 0.13560 & $0.01288(2)$ & $0.1886(3)$ & $0.06957(18)$ \\
\hline & & 0.13580 & $0.01027(2)$ & $0.1687(3)$ & $0.06771(18)$ \\
\hline & & 0.13600 & $0.00767(2)$ & $0.1463(3)$ & $0.06557(18)$ \\
\hline & & 0.13638 & $0.00272(2)$ & $0.0886(4)$ & $0.0603(2)$ \\
\hline
\end{tabular}

Table 17: Partially quenched average quark masses, pseudoscalar meson masses and decay constants in lattice units for $\beta=5.3$. 


\begin{tabular}{|c|c|c|c|c|c|}
\hline & $\kappa_{r}$ & $\kappa_{s}$ & $a m_{r s}$ & $a m_{\mathrm{PS}}$ & $F_{\mathrm{PS}}^{\text {bare }}$ \\
\hline \multirow{9}{*}{ N4 } & 0.13600 & 0.13600 & $0.02308(3)$ & $0.2172(6)$ & $0.0631(4)$ \\
\hline & 0.13615 & 0.13615 & $0.01885(3)$ & $0.1958(7)$ & $0.0605(4)$ \\
\hline & 0.13630 & 0.13630 & $0.01462(3)$ & $0.1723(8)$ & $0.0578(4)$ \\
\hline & 0.13645 & 0.13645 & $0.01040(3)$ & $0.1456(8)$ & $0.0548(4)$ \\
\hline & 0.13650 & 0.13600 & $0.01602(3)$ & $0.1805(8)$ & $0.0584(4)$ \\
\hline & & 0.13615 & $0.01391(3)$ & $0.1682(7)$ & $0.0572(4)$ \\
\hline & & 0.13630 & $0.01181(3)$ & $0.1550(8)$ & $0.0558(4)$ \\
\hline & & 0.13645 & $0.00970(3)$ & $0.1407(9)$ & $0.0543(4)$ \\
\hline & & 0.13650 & $0.00899(3)$ & $0.1357(8)$ & $0.0538(4)$ \\
\hline \multirow{9}{*}{ N5 } & 0.13600 & 0.13600 & $0.02265(3)$ & $0.2144(7)$ & $0.0622(4)$ \\
\hline & 0.13615 & 0.13615 & $0.01841(3)$ & $0.1927(7)$ & $0.0595(4)$ \\
\hline & 0.13630 & 0.13630 & $0.01419(3)$ & $0.1689(7)$ & $0.0566(4)$ \\
\hline & 0.13645 & 0.13645 & $0.00997(4)$ & $0.1417(8)$ & $0.0534(4)$ \\
\hline & 0.13660 & 0.13600 & $0.01417(4)$ & $0.1689(7)$ & $0.0558(4)$ \\
\hline & & 0.13615 & $0.01207(4)$ & $0.1559(7)$ & $0.0546(3)$ \\
\hline & & 0.13630 & $0.00996(4)$ & $0.1418(7)$ & $0.0532(3)$ \\
\hline & & 0.13645 & $0.00786(4)$ & $0.1262(8)$ & $0.0516(3)$ \\
\hline & & 0.13660 & $0.00575(4)$ & $0.1085(8)$ & $0.0499(4)$ \\
\hline \multirow{9}{*}{ N6 } & 0.13600 & 0.13600 & $0.022299(17)$ & $0.2099(6)$ & $0.0609(3)$ \\
\hline & 0.13615 & 0.13615 & $0.018062(17)$ & $0.1881(6)$ & $0.0581(3)$ \\
\hline & 0.13630 & 0.13630 & $0.013835(18)$ & $0.1642(6)$ & $0.0552(2)$ \\
\hline & 0.13645 & 0.13645 & $0.009619(19)$ & $0.1369(6)$ & $0.0519(3)$ \\
\hline & 0.13667 & 0.13600 & $0.01283(2)$ & $0.1586(6)$ & $0.0534(3)$ \\
\hline & & 0.13615 & $0.01073(2)$ & $0.1450(5)$ & $0.0522(3)$ \\
\hline & & 0.13630 & $0.00863(2)$ & $0.1301(5)$ & $0.0507(3)$ \\
\hline & & 0.13645 & $0.00653(2)$ & $0.1135(5)$ & $0.0491(3)$ \\
\hline & & 0.13667 & $0.00343(3)$ & $0.0834(7)$ & $0.0461(3)$ \\
\hline \multirow{9}{*}{$\mathrm{O} 7$} & 0.13600 & 0.13600 & $0.022117(10)$ & $0.2085(3)$ & $0.06040(14)$ \\
\hline & 0.13620 & 0.13620 & $0.016468(10)$ & $0.1789(3)$ & $0.05666(14)$ \\
\hline & 0.13640 & 0.13640 & $0.010837(12)$ & $0.1447(3)$ & $0.05241(14)$ \\
\hline & 0.13660 & 0.13660 & $0.005226(14)$ & $0.1012(3)$ & $0.04741(15)$ \\
\hline & 0.13671 & 0.13600 & $0.012084(13)$ & $0.1535(3)$ & $0.05225(18)$ \\
\hline & & 0.13620 & $0.009283(13)$ & $0.1345(3)$ & $0.05050(16)$ \\
\hline & & 0.13640 & $0.006483(13)$ & $0.1126(3)$ & $0.04841(16)$ \\
\hline & & 0.13660 & $0.003681(15)$ & $0.0855(3)$ & $0.04581(16)$ \\
\hline & & 0.13671 & $0.002130(15)$ & $0.0658(4)$ & $0.0440(2)$ \\
\hline
\end{tabular}

Table 18: Partially quenched average quark masses, pseudoscalar meson masses and decay constants in lattice units for $\beta=5.5$. 


\section{References}

[1] M. Lüscher, P. Weisz, and U. Wolff, A numerical method to compute the running coupling in asymptotically free theories, Nucl. Phys. B359 (1991) 221-243.

[2] M. Lüscher, R. Narayanan, P. Weisz, and U. Wolff, The Schrödinger functional: A renormalizable probe for nonabelian gauge theories, Nucl. Phys. B384 (1992) 168-228, [hep-lat/9207009].

[3] M. Lüscher, R. Sommer, P. Weisz, and U. Wolff, A precise determination of the running coupling in the SU(3) Yang-Mills theory, Nucl. Phys. B413 (1994) 481-502, [hep-lat/9309005].

[4] ALPHA Collaboration, A. Bode et al., First results on the running coupling in QCD with two massless flavors, Phys. Lett. B515 (2001) 49-56, [hep-lat/0105003].

[5] ALPHA Collaboration, M. Della Morte et al., Computation of the strong coupling in QCD with two dynamical flavours, Nucl. Phys. B713 (2005) 378-406, [hep-lat/0411025].

[6] ALPHA Collaboration, M. Della Morte et al., Non-perturbative quark mass renormalization in two-flavor QCD, Nucl. Phys. B729 (2005) 117-134, [hep-lat/0507035].

[7] S. Capitani, M. Della Morte, G. von Hippel, B. Knippschild, and H. Wittig, Scale setting via the $\Omega$ baryon mass, PoS LATTICE2011 (2011) 145, [arXiv:1110.6365].

[8] R. Sommer, A new way to set the energy scale in lattice gauge theories and its applications to the static force and $\alpha_{s}$ in SU(2) Yang-Mills theory, Nucl. Phys. B411 (1994) 839, [hep-lat/9310022].

[9] QCDSF-UKQCD Collaboration, M. Göckeler et al., Determination of light and strange quark masses from full lattice QCD, Phys.Lett. B639 (2006) 307-311, [hep-ph/0409312].

[10] K. G. Wilson, Confinement of quarks, Phys. Rev. D10 (1974) 2445-2459.

[11] B. Sheikholeslami and R. Wohlert, Improved continuum limit lattice action for QCD with Wilson fermions, Nucl. Phys. B259 (1985) 572. 
[12] M. Lüscher, S. Sint, R. Sommer, and P. Weisz, Chiral symmetry and $O(a)$ improvement in lattice QCD, Nucl. Phys. B478 (1996) 365-400, [hep-lat/9605038].

[13] ALPHA Collaboration, K. Jansen and R. Sommer, $O(a)$ improvement of lattice QCD with two flavors of Wilson quarks, Nucl. Phys. B530 (1998) 185-203, [hep-lat/9803017].

[14] M. Lüscher, Schwarz-preconditioned HMC algorithm for two-flavour lattice QCD, Comput. Phys. Commun. 165 (2005) 199, [hep-lat/0409106].

[15] M. Lüscher, Deflation acceleration of lattice QCD simulations, JHEP 12 (2007) 011, [arXiv:0710.5417].

[16] M. Lüscher, DD-HMC algorithm for two-flavour lattice QCD, http://luscher.web. cern. ch/luscher/DD-HMC/index.html.

[17] M. Lüscher, Solution of the Dirac equation in lattice QCD using a domain decomposition method, Comput. Phys. Commun. 156 (2004) 209-220, [hep-lat/0310048].

[18] M. Lüscher, Local coherence and deflation of the low quark modes in lattice QCD, JHEP 07 (2007) 081, [0706.2298].

[19] ALPHA Collaboration, S. Schaefer, R. Sommer, and F. Virotta, Critical slowing down and error analysis in lattice QCD simulations, Nucl.Phys. B845 (2011) 93-119, [arXiv:1009.5228].

[20] S. Duane, A. D. Kennedy, B. J. Pendleton, and D. Roweth, Hybrid Monte Carlo, Phys. Lett. B195 (1987) 216.

[21] M. Hasenbusch, Speeding up the Hybrid-Monte-Carlo algorithm for dynamical fermions, Phys. Lett. B519 (2001) 177-182, [hep-lat/0107019].

[22] M. Hasenbusch and K. Jansen, Speeding up lattice QCD simulations with clover-improved Wilson fermions, Nucl. Phys. B659 (2003) 299-320, [hep-lat/0211042].

[23] M. Marinkovic and S. Schaefer, Comparison of the mass preconditioned $H M C$ and the DD-HMC algorithm for two-flavour QCD, PoS

LATTICE2010 (2010) 031, [arXiv:1011.0911].

[24] ALPHA Collaboration, U. Wolff, Monte Carlo errors with less errors, Comput. Phys. Commun. 156 (2004) 143-153, [hep-lat/0306017]. 
[25] N. Madras and A. D. Sokal, The pivot algorithm: A highly efficient Monte Carlo method for the self-avoiding walk, J. Stat. Phys. 50 (1988) 109.

[26] S. Schaefer, R. Sommer, and F. Virotta, Investigating the critical slowing down of QCD simulations, PoS LAT2009 (2009) 032, [arXiv:0910.1465].

[27] M. Lüscher, Properties and uses of the Wilson flow in lattice QCD, JHEP 1008 (2010) 071, [arXiv: 1006 .4518].

[28] L. Del Debbio, L. Giusti, M. Lüscher, R. Petronzio, and N. Tantalo, QCD with light Wilson quarks on fine lattices. II: DD-HMC simulations and data analysis, JHEP 02 (2007) 082, [hep-lat/0701009].

[29] M. Della Morte, R. Hoffmann, and R. Sommer, Non-perturbative improvement of the axial current for dynamical Wilson fermions, JHEP 03 (2005) 029, [hep-lat/0503003].

[30] T. Bhattacharya, R. Gupta, W. Lee, S. R. Sharpe, and J. M. Wu, Improved bilinears in lattice QCD with non-degenerate quarks, Phys.Rev. D73 (2006) 034504, [hep-lat/0511014].

[31] S. Sint and P. Weisz, Further results on $O(a)$ improved lattice QCD to one loop order of perturbation theory, Nucl. Phys. B502 (1997) 251, [hep-lat/9704001].

[32] M. Della Morte, R. Sommer, and S. Takeda, On cutoff effects in lattice QCD from short to long distances, Phys.Lett. B672 (2009) 407-412, [arXiv:0807.1120].

[33] R. Sommer, Leptonic decays of B and D mesons, Nucl.Phys.Proc.Suppl. 42 (1995) 186-193, [hep-lat/9411024].

[34] UKQCD Collaboration, M. Foster and C. Michael, Quark mass dependence of hadron masses from lattice QCD, Phys. Rev. D59 (1999) 074503, [hep-lat/9810021].

[35] O. Bär and M. Golterman, in preparation.

[36] M. Donnellan, F. Knechtli, B. Leder, and R. Sommer, Determination of the Static Potential with Dynamical Fermions, Nucl.Phys. B849 (2011) 45-63, [arXiv: 1012.3037]. 
[37] A. Hasenfratz and F. Knechtli, Flavor symmetry and the static potential with hypercubic blocking, Phys. Rev. D64 (2001) 034504, [hep-lat/0103029].

[38] M. Della Morte, A. Shindler, and R. Sommer, On lattice actions for static quarks, JHEP 08 (2005) 051, [hep-lat/0506008].

[39] F. Knechtli and B. Leder, The shape of the static potential with dynamical fermions, arXiv:1112.1246.

[40] QCDSF-UKQCD Collaboration, D. Brömmel et al., The Pion form-factor from lattice QCD with two dynamical flavours, Eur.Phys.J. C51 (2007) 335-345, [hep-lat/0608021].

[41] ALPHA Collaboration, B. Leder and F. Knechtli, Scale $r_{0}$ and the static potential from the CLS lattices, PoS LATTICE2010 (2010) 233, [arXiv: 1012.1141].

[42] G. Colangelo et al., Review of lattice results concerning low energy particle physics, Eur.Phys.J. C71 (2011) 1695, [arXiv:1011.4408].

[43] A. Roessl, Pion kaon scattering near the threshold in chiral SU(2) perturbation theory, Nucl.Phys. B555 (1999) 507-539, [hep-ph/9904230].

[44] RBC-UKQCD Collaboration, C. Allton et al., Physical Results from $2+1$ Flavor Domain Wall QCD and SU(2) Chiral Perturbation Theory, Phys.Rev. D78 (2008) 114509, [arXiv:0804.0473].

[45] S. R. Sharpe, Enhanced chiral logarithms in partially quenched QCD, Phys.Rev. D56 (1997) 7052-7058, [hep-lat/9707018].

[46] P. Fritzsch, J. Heitger, and N. Tantalo, Non-perturbative improvement of quark mass renormalization in two-flavour lattice QCD, JHEP 1008 (2010) 074, [arXiv: 1004.3978].

[47] B. Blossier, M. Della Morte, P. Fritzsch, N. Garron, J. Heitger, et al., Parameters of Heavy Quark Effective Theory from $N f=2$ lattice $Q C D$, arXiv: 1203.6516.

[48] S. Necco and R. Sommer, The $N_{\mathrm{f}}=0$ heavy quark potential from short to intermediate distances, Nucl. Phys. B622 (2002) 328-346, [hep-lat/0108008]. 
[49] R. Sommer, Non-perturbative QCD: Renormalization, O(a)-improvement and matching to Heavy Quark Effective Theory, hep-lat/0611020.

[50] S. Sint and R. Sommer, The running coupling from the QCD Schrödinger functional: A one loop analysis, Nucl. Phys. B465 (1996) 71-98, [hep-lat/9508012].

[51] ALPHA Collaboration, S. Capitani, M. Lüscher, R. Sommer, and H. Wittig, Non-perturbative quark mass renormalization in quenched lattice QCD, Nucl. Phys. B544 (1999) 669, [hep-lat/9810063].

[52] ALPHA Collaboration, S. Sint and P. Weisz, The Running quark mass in the SF scheme and its two loop anomalous dimension, Nucl. Phys. B545 (1999) 529, [hep-lat/9808013].

[53] S. Bethke, A. H. Hoang, S. Kluth, J. Schieck, I. W. Stewart, et al., Workshop on Precision Measurements of $\alpha_{s}$, arXiv:1110.0016.

[54] ALPHA Collaboration, J. Garden, J. Heitger, R. Sommer, and H. Wittig, Precision computation of the strange quark's mass in quenched QCD, Nucl. Phys. B571 (2000) 237-256, [hep-lat/9906013].

[55] S. Alekhin, J. Blümlein, and S. Moch, Parton distribution functions and benchmark cross sections at NNLO, arXiv:1202.2281.

[56] J. C. Sexton and D. H. Weingarten, Hamiltonian evolution for the hybrid Monte Carlo algorithm, Nucl. Phys. B380 (1992) 665-678.

[57] I. P. Omelyan, I. M. Mryglod, and R. Folk, Symplectic analytically integrable decomposition algorithms: classification, derivation, and application to molecular dynamics, quantum and celestial mechanics simulations, Computer Physics Communications 151 (2003), no. 3272 - 314.

[58] M. Della Morte, R. Hoffmann, F. Knechtli, R. Sommer, and U. Wolff, Non-perturbative renormalization of the axial current with dynamical Wilson fermions, JHEP 07 (2005) 007, [hep-lat/0505026].

[59] ALPHA Collaboration, M. Guagnelli et al., Non-perturbative results for the coefficients $b_{m}$ and $b_{A}-b_{P}$ in $O(a)$ improved lattice $Q C D$, Nucl. Phys. B595 (2001) 44-62, [hep-lat/0009021]. 